\title{
Evolución de la perspectiva responsable en la Ciencia e Innovación (RRI) en España: un análisis de los documentos de gobernanza estratégica
}

Paula Otero-Hermida

Diciembre 2020

Para citar este documento: Paula Otero-Hermida (2020). Evolución de la perspectiva responsable en la Ciencia e Innovación en España (RRI): un análisis de los documentos de gobernanza estratégica. December 2020, Working paper, SUPER_MoRRI project www.supermorri.eu 
Este trabajo se ha desarrollado en el marco del proyecto SUPER MoRRI - Scientific understanding and provision of an enhanced and robust monitoring system for RRI

El proyecto ha recibido financiación del programa de investigación e innovación Horizonte 2020 de la Unión Europea en virtud del acuerdo de subvención No 824671. Las opiniones expresadas en el presente documento reflejan únicamente la opinión de los autores y en ningún caso las opiniones de la Comisión Europea. La Comisión Europea no es responsable del uso que pueda hacerse de la información que contiene. 


\section{Contenido}

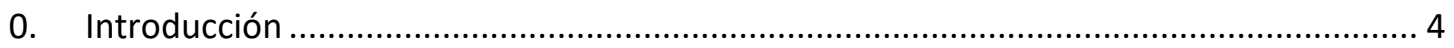

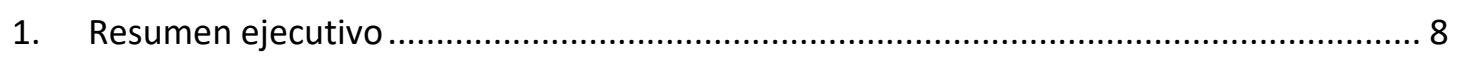

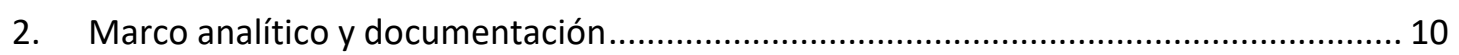

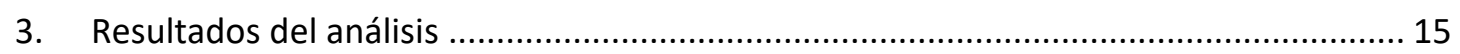

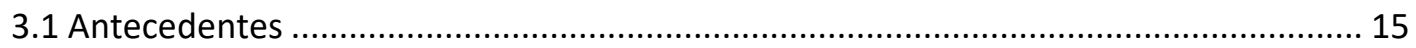

3.2 El sistema de gobierno y gobernanza de la Ciencia y la Innovación .............................. 15

3.3 Definición de prioridades y participación pública....................................................... 18

3.4 Estrategias y Planes de Ciencia, Tecnología e Innovación: aproximaciones RRI ........... 19

3.5 Monitorización: El Sistema de Información sobre Ciencia, Tecnología e Innovación... 28

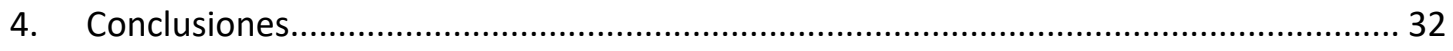

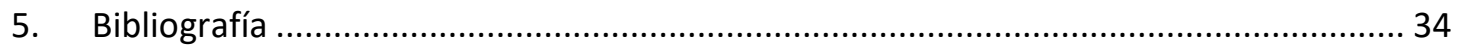




\section{Introducción}

Las perspectivas responsables en Ciencia, Innovación y Tecnología están cada vez más presentes en el debate público y político, al igual que lo están las preguntas a las que pretenden dar respuesta: ¿los avances científicos y tecnológicos están siendo beneficiosos, de manera global? ¿cuáles están siendo perjudiciales y porqué se está produciendo esta situación? ¿a quién benefician, y quién se está quedando fuera? ¿cómo hacer compatible el avance con "una vida auténtica en la tierra," también para las generaciones futuras, como lo definía Hans Jonas en los años 70 (Jonas, 1979). Estas perspectivas y preguntas se hacen cada vez más relevantes, a medida que la ciencia y la innovación se vuelven más centrales en la sociedad actual.

En el marco de los con los estudios de ciencia y tecnología (STS) se ha desarrollado un área que estudia específicamente estas cuestiones, rotulada en sus siglas inglesas como RRI (Responsible Research and Innovation). Su punto de partida se sitúa en torno a 2010, coincidiendo con el impulso de la Comisión Europea, que desde entonces promueve políticas específicas bajo esta denominación.

Uno de los factores que podría citarse en la aparición de la RRI está relacionado con la combinación entre altas expectativas incumplidas, así como los fallos y las consecuencias inesperadas de la ciencia (Owen, Bessant \& Heintz, 2013), muchos de los cuales han sido altamente discutidos en los últimos cincuenta años. Desde la meta prometida, pero no alcanzada, del fin de la pobreza, al impacto negativo en el medioambiente, muchas veces no reversible en el curso de una vida humana y por tanto constitutivo de un legado tóxico a nuestros descendientes. A las expectativas incumplidas se suma la complejidad que genera su gestión. La ciencia y la innovación generan certezas, pero sus implicaciones en la vida práctica, una vez se encuentran en el mundo real, son muchas veces impredecibles. Se cita por ello que el gran desafío es la gestión de la incertidumbre y la complejidad, que no necesariamente se están reduciendo sino aumentando ante la aparición creciente de nuevas tecnologías. Ante estos desafíos, las perspectivas RRI proponen un enfoque que va más allá de la tradicional gestión del riesgo. Estas corrientes parten no obstante de un gran número de iniciativas y trabajo en décadas precedentes para acercar las ciencias duras a las ciencias sociales y motivar el debate público sobre cuestiones sensibles, introduciendo la consideración de aspectos legales y éticos, como las iniciativas ELSA y ELSI en el estudio y mapeo del genoma humano, tanto en Estados Unidos como en Europa. Las nuevas perspectivas RRI sin embargo asumen que hay cuestiones que no pueden ser controladas a posteriori, y por lo tanto se alejan de perspectivas éticas consecuencialistas, que asocian responsabilidades a las evidencias sobre lo ya acaecido. Desde las perspectivas RRI el proceso de responsabilidad comenzaría antes, situándose en la concepción de los proyectos científicos e innovadores, con la mirada enfocada en las posibles consecuencias e impactos futuros a través del ejercicio de la reflexividad (Owen et al, 2013; Owen et al, 2012) (Gribaum \& Grooves, 2013).

Las perspectivas RRI muestran especial atención a los "impactos correctos" en la ciencia y la innovación, incluidas las contribuciones "socialmente deseables", que se anclarían en puntos normativos específicos, como los principios de igualdad expuestos en el Tratado de la Unión Europea (Von Schomberg, 2013, 2014). Tal como prevén las instituciones europeas, la RRI debe centrarse en acercar la ciencia y la innovación a la sociedad, con la participación de diferentes agentes, realizando un esfuerzo renovado en la inclusión de actores sociales no tradicionales en el ámbito de la ciencia e innovación, es decir, más allá de los actores académicos e industriales. Utilizando términos como "co-creación", "ciencia abierta" o "compromiso público", las instituciones de la Unión Europea están señalando la necesidad de hacer accesible la ciencia, con más personas participando en su desarrollo, y por lo tanto inciden en la necesidad de una nueva gobernanza. Estos aspectos relativos a la participación pública son especialmente visibles 
en el nuevo período de programación Horizonte Europa ${ }^{1}$. Si bien el concepto RRI no aparece como lo hizo en el anterior período de programación, en los nuevos documentos estratégicos de la Comisión Europea sí encontramos los aspectos relacionados con esta nueva perspectiva normativa en las políticas públicas, detallados a continuación.

En los documentos iniciales relativos a RRI (Comisión Europea, 2012, 2014), la UE sentó las bases de una aproximación por áreas, específicamente las siguientes: gobernanza, participación pública, ética, educación para la ciencia, acceso abierto- actualmente ciencia abierta- e igualdad de género. A estas propuestas temáticas se suman propuestas académicas que han sido integradas en numerosos proyectos financiados con fondos $\mathrm{H} 2020$, perfiladas en dimensiones: anticipación, reflexividad, inclusividad, apertura, capacidad de respuesta (Owen et al, 2013; Owen et al, 2012; Stilgoe et al, 2013). Así mismo, los marcos incluyen aspectos clave en el desarrollo de los estudios de ciencia e innovación, como la necesidad de multidisciplinariedad e interdisciplinariedad para ofrecer conocimiento científico útil para la sociedad ${ }^{2}$.

Desde estos delineamientos básicos iniciales en cuanto a áreas de intervención y dimensiones, diversas iniciativas y preguntas de investigación han sido desarrolladas. Muchas de ellas, focalizadas en la evaluación y monitorización de la integración de las líneas RRI en las políticas de ciencia e innovación, como el proyecto del que parte este trabajo, así como otras enfocadas en áreas concretas o en el desarrollo RRI territorial- regional y local-, como los proyectos con financiación H2O20 dentro del programa Science for and with society-SWAFS-14.

Diversos proyectos están por lo tanto estudiando la aplicación práctica de estos principios a las políticas de innovación y ciencia, también desde el punto de vista de las organizaciones que innovan o investigan, observando potencialidades, beneficios de la práctica y posibles barreras. El conocimiento de las similitudes y diferencias observadas entre los países abre vías para aprender y compartir nuevas prácticas, procedimientos y entendimientos del concepto de responsabilidad, formas de pensar en una comunidad profesional formada por administradores, encargados de la formulación de políticas y un público que comparte sus nociones básicas derivadas de experiencias pasadas financiadas por la UE (Meijlgaard et al, 2018).

En el presente documento, el foco es analizar el marco de partida de la institucionalización en España de las políticas RRI y sus aspectos relacionados- ética, participación pública, multidisciplinariedad, reflexividad, etc-. Para ello, se presentan los resultados preliminares del análisis de los principales documentos de gobernanza españoles: las estrategias de ciencia, tecnología e innovación correspondiente al período 2014-2020, así como la nueva estrategia 2021-2027, presentada en el mes de septiembre de 2020. También sus planes asociados y otros documentos relacionados, como aquellos destinados a la planificación anual de la financiación. En el apartado siguiente, puede encontrarse más información sobre los documentos y el marco analítico. Continuaremos detallando aquí el marco general del trabajo.

El proyecto SUPER_MoRI, dedicado a la construcción de un marco para las políticas de RRI y su monitorización, está desarrollando un estudio con el fin de comparar el trabajo en la materia y los contextos de a) instituciones dedicadas a la financiación de la ciencia y la innovación b) instituciones que realizan actividades científicas e innovadoras. Para ello se ha construido una red de representantes en 30 países europeos y se desarrollarán casos de estudio por país

\footnotetext{
${ }^{1}$ Este enfoque puede apreciarse en los diferentes documentos que delinean la estructura del programa marco, citados en la sección de marco analítico y documentación.

${ }^{2}$ Mayor detalle con respecto a estos aspectos en la sección relativa a marco analítico del presente documento.
} 
(Country Correspondents Network-CCN). El presente trabajo constituye la sección de análisis documental del caso español, que será posteriormente completado con entrevistas.

Las relaciones entre políticas públicas europeas y los contextos nacionales suelen son objeto de estudio, ante la necesidad de adaptación que éstas conllevan. Al igual que los productos de la ciencia y la innovación, las políticas tienen un recorrido desde su ideación inicial a su aplicación, especialmente al insertarse en diferentes entornos, con prácticas y culturas institucionales diversas. Cabe por tanto preguntarse qué similitudes y evoluciones encontramos con respecto al marco europeo RRI en nuestro país, qué elementos han sido destacados o inhibidos, o qué aspectos relevantes han sido introducidos por constituir problemáticas específicas de nuestro contexto, entre otras posibles preguntas.

Los documentos analizados se sitúan en el nivel estratégico o de mandato en los marcos de análisis de las políticas públicas utilizados. Son, por tanto, elementos centrales en el diseño de las políticas. Definen qué se quiere hacer, cuáles son sus principios, sus objetivos y finalidades últimas, los elementos de los cuáles se compone (estrategias), así como se trazan líneas para su implementación futura (planes). Este análisis, por lo tanto, no permite observar impactos o resultados de las políticas, sino que el foco está en qué entendemos por la política misma.

En este sentido, cabe resaltar la importancia de la gobernanza en la política analizada, y por tanto, la especial relevancia de atender a los marcos de gobernanza. Por supuesto, la gobernanza es clave en todas las políticas públicas, si bien, en este caso como se indicaba, la RRI se perfila como un nuevo modelo de gobernanza para las políticas de ciencia e innovación. El principal pilar del cambio es la citada participación pública más allá de los actores tradicionales de la política. Sin embargo, incluso en los países que se consideran más avanzados en cuanto a la participación ciudadana, se ha observado una falta de conexión entre esos procesos participativos de carácter local o específico en cuanto a ámbitos temáticos y la adopción de decisiones en materia de gobernanza. Las conclusiones del proyecto STAGE incidían en que las innovaciones en la participación exigían que se prestara más atención al contexto de la gobernanza y a la posibilidad de incorporar la deliberación en él (Hagendijk \& Irwin, 2006). También se ha observado una tendencia a centrarse en soluciones técnicas o en problemas que se consideran más fáciles de resolver (Nielsen et al. 2016:59). Además de estos problemas relativos a la definición de las políticas, la cartografía de la gobernanza de facto implica comprender, al más alto nivel, el establecimiento de programas que a menudo sirven a imaginarios sociotécnicos particulares, que pueden ser difíciles de dilucidar (Jasanoff, 2015). Por ello, una de las mayores contribuciones de los estudios sobre ciencia y tecnología y de los estudios sobre políticas científicas ha sido revelar las formas en que las trayectorias de la ciencia y la tecnología no están predeterminadas, sino que se rigen, a menudo, por supuestos no explícitos.

La necesidad de estudio de los marcos de las políticas es especialmente destacada por las ramas social-constructivistas, así como han sido abrazadas por la corriente institucionalista que predomina en los enfoques politológicos que trabajan con políticas normativas como la igualdad de género (Mackay et al, 2009). Más allá de corrientes específicas, cabe destacar lo fundamental que resulta el análisis de los marcos, dados los fenómenos de inclusión/exclusión de aspectos que finalmente resultan en una política con un carácter $u$ otro. Adelantando las conclusiones, cabe señalar la exclusión observada en el análisis realizado, de las menciones a las posibles consecuencias indeseadas de la innovación y su reflexión sobre ellas. Este hallazgo puede observarse también como una asunción implícita de la innovación como algo netamente positivo, un ideal a convertirse en elemento transversal en todas las políticas públicas, sin que se hallen menciones a uno de los principales motivos por los cuales las políticas de RRI hicieron su aparición, los posibles riesgos, incertidumbres y consecuencias negativas también generadas por los procesos innovadores que afectan a la sociedad. El principal mecanismo por el que se 
produce esta situación podría ser el conocido fenómeno de isomorfismo institucional (DiMaggio \& Powell, 2000) por el cual se adoptan políticas procedentes con frecuencia de niveles administrativos superiores sin realizar cambios. Podría ser el caso de las políticas científicas y de innovación RRI españolas, que han sido observadas como una asimilación, más que adaptación, de las políticas europeas al propio contexto. No se han detectado elementos o aproximaciones propias en el marco español de la política, salvando las menciones a la precariedad. En el nuevo período de programación europea se observa también una retracción de la consideración explícita de posibles consecuencias indeseadas o inesperadas de la innovación, mientras se ha enfatizado la necesidad de participación pública y de actores sociales en la gobernanza de las políticas de ciencia e innovación. Esta misma evolución ha sido observada en los documentos analizados, como se detalla en lo expuesto en este documento.

Para concluir esta introducción, se presentan las secciones del estudio. En primer lugar, se puede encontrar a continuación un resumen ejecutivo de los principales hallazgos. Prosiguiendo, la siguiente sección presenta el marco analítico y las referencias documentales. En tercer lugar, se sitúan los resultados o hallazgos del análisis, precedidos de una introducción al Sistema de Ciencia e Innovación Español, para seguidamente centrarse en el análisis respecto a la aproximación RRI de las estrategias y planes que constituyen los principales documentos de gobernanza. Finalmente pueden encontrarse las conclusiones y la bibliografía. 


\section{Resumen ejecutivo}

El Sistema Español de Ciencia, Tecnología e Innovación se caracteriza por un marco de gobernanza multinivel y la impronta de los procesos de europeización que han canalizado los fondos europeos. Los documentos oficiales analizados explicitan claramente estos aspectos en su descripción del sistema. Desde el año 2013 la gobernanza se adapta a los procedimientos de management público propuestos por la UE. Estos documentos indican las distintas prioridades, objetivos y estructuras de financiación: a) las Estrategias Españolas de Ciencia y Tecnología definen objetivos generales y el sentido y ordenación general del sistema. B) De las estrategias derivan los Planes Estatales que concretan objetivos y sus instrumentos cada tres años. A su vez, los planes estatales se implementan a través de planes de actuación anuales, calendarizando las diversas convocatorias de fondos y la inversión pública adscrita a cada uno de ellos. Partiendo de este marco, la Agencia Estatal de Investigación gestiona el grueso del presupuesto y de las convocatorias para ciencia, estimando la evaluación por pares de 32.000 solicitudes y la realización de 17.000 evaluaciones en 2020. La gestión de los fondos de Innovación la desarrolla otra agencia, CDTI, mientras otras convocatorias son gestionadas por otros Ministerios como Universidades, Defensa, la Fundación FECYT o el Instituto de Salud Carlos III en la acción estratégica Salud.

Respecto a RRI, el marco de referencia, es el siguiente. La estrategia 2013-2020 fue gestada durante la crisis económica global y un gobierno situado a la derecha, mientras la reciente estrategia 2021-2027 ha sido redactada bajo un gobierno situado a la izquierda y una crisis pandémica y socioeconómica. La Estrategia es así mismo la Estrategia de Especialización Inteligente (S3) estatal, marco para las S3 regionales. Las referencias a RRI en la primera estrategia son prácticamente inexistentes, estando orientada a mejorar la competitividad y la transferencia al mercado, si bien en su último plan de actuación 2017-2020 se observa la influencia de la política europea de RRI, sin observarse adaptaciones específicas o peculiaridades nacionales en sus menciones a género, ciencia accesible o impacto social. En este nueva Estrategia que alcanza hasta 2027, la propuesta relativa al impacto o marco social está presente en objetivos, resultados esperados, principios y elementos transversales. El objetivo ha cambiado, pretendiendo progresar hacia "una economía basada en una tecnología baja en carbono, digitalizada, sostenible y socialmente responsable". Los Objetivos de Desarrollo del Milenio forman parte del marco. Género, ciencia abierta y percepción social de la ciencia están presentes en distintas áreas de la estrategia, así como en el análisis DAFO. Específicamente, se cita la precariedad del personal, especialmente el más joven, como una problemática del sistema. Las líneas temáticas prioritarias se enmarcan en lo definido en Horizonte Europa: 1. Salud,2. Cultura, Creatividad y Sociedad Inclusiva, 3. Seguridad Civil para la Sociedad, 4. Mundo digital, Industria, Espacio y Defensa, 5. Clima, energía y movilidad, 6. Alimentación, Bioeconomía, Recursos Naturales y Medio Ambiente. Se espera que esta estructura "incentivará la multidisciplinariedad que permitirá el desarrollo de misiones científicas y el abordaje proyectos tractores de los ODS". Así mismo, el nuevo sistema de gobernanza de la estrategia cita un enfoque Cuatruple Hèlix, e incluye dos asociaciones civiles en su comité de seguimiento.

Por último, cabe destacar que el conjunto de los documentos analizados muestra una imagen netamente positiva de la ciencia y la innovación, sin alusiones a consecuencias indeseadas o inesperadas, aspecto clave en el marco RRI, no obstante. Así mismo, aludiendo al impacto de la pandemia, la estrategia 2021-2027 sitúa la ciencia e innovación en un nuevo lugar preeminente y transversal en el conjunto de las políticas públicas y la toma de decisiones, aspecto que no estaba presente en los anteriores documentos de gobernanza. 
Con respecto a la monitorización, se alude a que se desarrollará un sistema de evaluación que incluya indicadores cuantitativos y cualitativos, pero éstos últimos no están presentes en la estrategia. En la tabla de indicadores prioritarios sólo está presente un indicador de género (mujeres investigadoras). Sin embargo, en el Anexo es posible encontrar indicadores de seguimiento de objetivos relativos a ciencia y sociedad, mujeres empleadas en I+D, comunicación para la ciencia y multidisciplinariedad. Son ausencia destacada: indicadores en el ámbito empresarial e indicadores de ciencia abierta, pese a lo presente que está en el documento. 


\section{Marco analítico y documentación}

Como se citaba anteriormente, las preguntas de investigación y elementos analíticos son parte de un estudio más amplio, y han sido definidos de forma común por el equipo del proyecto SUPER_MoRRI para la red de representantes, para cada caso de estudio que constituyen los países europeos incluidos (30). La red de representantes también ha participado en afinar las preguntas y marco analítico a través de dos sesiones de trabajo realizadas entre finales de noviembre y principios de diciembre de 2020. El marco analítico completo puede ser consultado en inglés en el protocolo del estudio desarrollado para la red, disponible para su acceso público en la plataforma $\mathrm{OSF}^{3}$. Se muestran a continuación los elementos más relevantes, utilizados en el presente documento que muestra los resultados del caso español.

La investigación está destinada s a conocer más en relación a los siguientes aspectos:

- ¿Cómo se apoya el desarrollo de prácticas y culturas de investigación responsables mediante el diseño y la aplicación de escenarios prioritarios?

- ¿Cómo se define el desarrollo de prácticas y culturas de investigación responsables mediante el diseño y la aplicación de instrumentos de financiación?

- ¿Cómo se apoya la investigación el desarrollo de prácticas y culturas de investigación responsables a través del diseño y la implementación de evaluaciones de financiación de la investigación?

En el caso del presente trabajo, se analizan las bases que los documentos de gobernanza estratégica sientan para favorecer los citados aspectos: priorización de aspectos estratégicos, desarrollo de instrumentos de financiación que favorezcan la RRI, inclusión de elementos de evaluación que valoren dichos aspectos. También, considerando la relevancia en cuanto a gobernanza estratégica, se analizan los elementos correspondientes a la monitorización.

En la siguiente tabla se pueden observar de forma más detallada a qué aspectos se refiere el trabajo.

Tabla 1. Definiciones del estudio

\begin{tabular}{|l|l|}
\hline \multirow{2}{*}{ Prácticas de investigación responsable } & Se refiere a todos los aspectos de la investigación \\
\cline { 2 - 2 } & $\begin{array}{l}\text { Aspectos relativos a cómo se diseña la } \\
\text { investigación: } \\
\text { análisis de género; preinscripción; reflexión sobre } \\
\text { las posibles consecuencias negativas; ciudadanía- } \\
\text { ciencia; socios no académicos; consulta con los } \\
\text { interesados sobre cuestiones de investigación o } \\
\text { métodos; co-creación de problemas, preguntas y } \\
\text { enfoques de investigación con diversos socios; etc. } \\
\text { Cómo se implementa un diseño de investigación: }\end{array}$ \\
\hline
\end{tabular}

\footnotetext{
${ }^{3}$ Protocol for the Country Correspondent Network, Study of Research Funding Organisations (CCN-RFO), V1 16 November 2020. https://osf.io/5ufzil Área de acceso: SUPER_MoRRI project, Working Pakage 2Data collection (WP2), CCN study.
} 


\begin{tabular}{|c|c|}
\hline & $\begin{array}{l}\text { apertura; reproducibilidad; integridad de la } \\
\text { investigación; conducta ética; transparencia en } \\
\text { cuanto a modificaciones de diseño, etc. } \\
\text { Cómo se informa y se difunde la investigación: } \\
\text { Depósito de datos abiertos FAIR; no hay fraude en } \\
\text { la publicación; no hay piratería informática; } \\
\text { difusión a participantes e interesados; } \\
\text { comunicación al público; etc. }\end{array}$ \\
\hline \multirow[t]{6}{*}{ Culturas de investigación responsable } & $\begin{array}{l}\text { Se refiere a todos los aspectos del entorno de } \\
\text { investigación }\end{array}$ \\
\hline & $\begin{array}{l}\text { Formación de investigadores: } \\
\text { ciencia abierta; datos abiertos de FAIR; principios } \\
\text { de anticipación, reflexión, inclusión y respuesta } \\
\text { (RRI); herramienta de pensamiento de } \\
\text { preparación de la sociedad; integridad y ética de } \\
\text { la investigación; } \\
\text { sensibilidad cultural; diseños de investigación } \\
\text { comprometidos; etc. }\end{array}$ \\
\hline & $\begin{array}{l}\text { Evaluación de la investigación y los } \\
\text { investigadores: } \\
\text { Declaración sobre la evaluación de la } \\
\text { investigación (DORA); Reconocimiento y } \\
\text { recompensa por las contribuciones científicas de } \\
\text { los investigadores y su sociedad contribuciones: } \\
\text { empleo; promoción; evaluación; valoración de } \\
\text { propuestas de subvención; formatos alternativos } \\
\text { de currículum vitae y criterios de evaluación de } \\
\text { diversos tipos; etc. }\end{array}$ \\
\hline & $\begin{array}{l}\text { Reconocimiento y recompensa de las } \\
\text { contribuciones interdisciplinarias de los } \\
\text { investigadores: } \\
\text { evaluación; valoración de propuestas de } \\
\text { subvención; Valoración compartida y sistémica de } \\
\text { las prácticas de investigación responsables Apoyo } \\
\text { al desarrollo de competencias profesionales } \\
\text { responsables por parte de los dirigentes a todos } \\
\text { los niveles de la organización formal e informal de } \\
\text { la investigación: grupos; especializaciones; } \\
\text { comunidades epistémicas; campos científicos; }\end{array}$ \\
\hline & $\begin{array}{l}\text { Apoyo formal (incentivos y recompensas) para las } \\
\text { carreras de investigación que hacen que tanto la } \\
\text { ciencia y las contribuciones de la sociedad: } \\
\text { universidades; organizaciones de investigación del } \\
\text { sector público; organización de financiación de la } \\
\text { investigación; organismos de acreditación; } \\
\text { marcos de evaluación; etc. }\end{array}$ \\
\hline & $\begin{array}{l}\text { Apoyo formal (procedimientos de organización) a } \\
\text { las culturas de investigación responsables: } \\
\text { igualdad de género en los paneles de } \\
\text { contratación, comités de ética, comités de } \\
\text { gestión; etc. }\end{array}$ \\
\hline
\end{tabular}


Fuente: Protocol for the Country Correspondent Network, Study of Research Funding Organisations (CCNRFO), V1 16 November 2020 SUPER_MoRRI project. Traducción de la autora

Seguidamente se encuentra el esquema inicial de codificación utilizado para el análisis documental.

\section{Tabla 2. Esquema de codificación}

\begin{tabular}{|c|c|c|c|c|}
\hline Mecanismo & Pregunta & Sub-pregunta & Variables & Dimensiones \\
\hline \multirow[t]{4}{*}{$\begin{array}{l}\text { Definición de } \\
\text { prioridades }\end{array}$} & \multirow[t]{3}{*}{$\begin{array}{l}\text { ¿Cómo se definen } \\
\text { las prioridades? }\end{array}$} & $\begin{array}{lr}\text { De dónde vienen } \\
\text { las visiones para } \\
\text { sentar } & \text { las } \\
\text { prioridades? } & \\
\end{array}$ & $\begin{array}{l}\text { Autoridad } \\
\text { Fuentes externas } \\
\text { Co-creación }\end{array}$ & $\begin{array}{l}\text { UE-nacional, Objetivos Desarrollo } \\
\text { Milenio (ODS), redes de pares, } \\
\text { grupos de interés y participación }\end{array}$ \\
\hline & & $\begin{array}{l}\text { Qué grupos de } \\
\text { interés participan } \\
\text { en la definición de } \\
\text { prioridades? }\end{array}$ & $\begin{array}{l}\text { Tipo } \\
\text { Interés } \\
\text { Nivel }\end{array}$ & Sector público, privado, otros \\
\hline & & $\begin{array}{lr}\text { Cómo } & \text { las } \\
\text { prioridades } & \text { se } \\
\text { coproducen? } & \\
\end{array}$ & Proceso & \\
\hline & $\begin{array}{l}\text { ¿Están las } \\
\text { prioridades } \\
\text { relacionadas con } \\
\text { otras políticas } \\
\text { nacionales? }\end{array}$ & & $\begin{array}{l}\text { Áreas de las políticas: } \\
\text { salud, bienestar } \\
\text { social, educación, } \\
\text { otras }\end{array}$ & $\begin{array}{l}\text { Políticas: } \quad \text { Estrategias de } \\
\text { Especialización } \\
\text { de inteligente, políticas } \\
\text { innación... }\end{array}$ \\
\hline \multirow[t]{10}{*}{$\begin{array}{l}\text { Instrumentos de } \\
\text { financiación }\end{array}$} & \multirow{3}{*}{$\begin{array}{lr}\text { ¿Cómo } & \text { se } \\
\text { desarrollan } & \text { los } \\
\text { instrumentos } & \text { de } \\
\text { financiación? } & \end{array}$} & $\begin{array}{l}\text { ¿Qué grupos de } \\
\text { interés } \\
\text { contribuyen? }\end{array}$ & Tipo, interés & Público, privado, otros \\
\hline & & $\begin{array}{l}\text { ¿Cómo se crean los } \\
\text { instrumentos de } \\
\text { financiación? }\end{array}$ & Proceso & \\
\hline & & $\begin{array}{lr}\text { ¿Qué } & \text { grupos } \\
\text { podrían } & \text { ser } \\
\text { sumados } & \text { al } \\
\text { proceso? } & \\
\end{array}$ & Tipo, interés & Público, privado, otros \\
\hline & \multirow[t]{7}{*}{$\begin{array}{l}\text { Contienen los } \\
\text { instrumentos de } \\
\text { financiación } \\
\text { incentivos para } \\
\text { una investigación } \\
\text { más responsable? }\end{array}$} & $\begin{array}{l}\text { Diseños de } \\
\text { investigación } \\
\text { participativa con } \\
\text { ciudadanía y } \\
\text { grupos de interés }\end{array}$ & $\begin{array}{l}\text { Socios no } \\
\text { académicos, públicos, } \\
\text { beneficiarios, } \\
\text { innovadores }\end{array}$ & $\begin{array}{l}\text { Estadio de la investigación y ciclo de } \\
\text { innovación: cocreación, ciencia } \\
\text { ciudadana, educación para la ciencia. }\end{array}$ \\
\hline & & $\begin{array}{l}\text { Perspectiva de } \\
\text { género }\end{array}$ & $\begin{array}{l}\text { Equipos con equilibrio } \\
\text { de género } \\
\text { Perspectiva de género } \\
\text { en la investigación } \\
\text { otros }\end{array}$ & \\
\hline & & Ciencia abierta & $\begin{array}{l}\text { Datos abiertos; } \\
\text { Acceso abierto; } \\
\text { Software abierto; } \\
\text { flujos de trabajo } \\
\text { abiertos; } \\
\text { etc. }\end{array}$ & $\begin{array}{l}\text { Depósito de conjuntos de datos; } \\
\text { Apoyo al intercambio de datos; } \\
\text { publicaciones de acceso abierto; } \\
\text { Uso de servidores de preimpresión; } \\
\text { etc. }\end{array}$ \\
\hline & & Marco AIRR & $\begin{array}{l}\text { Anticipación } \\
\text { Reflexión }\end{array}$ & $\begin{array}{l}\text { Positivo } \\
\text { Negativo } \\
\text { Identificación de posibles } \\
\text { beneficiarios y grupos vulnerables }\end{array}$ \\
\hline & & $\begin{array}{l}\text { Ética e integridad } \\
\text { en la investigación }\end{array}$ & $\begin{array}{l}\text { Valores personales } \\
\text { Proceso de } \\
\text { investigación }\end{array}$ & $\begin{array}{l}\text { Honestidad, cuidado y respeto; } \\
\text { rigor, transparencia y comunicación } \\
\text { abierta, responsabilidad, privacidad }\end{array}$ \\
\hline & & Vías de innovación & Grupos interesados & $\begin{array}{l}\begin{array}{l}\text { Actividades participativas y de } \\
\text { difusión }\end{array} \\
\end{array}$ \\
\hline & & Ciencia ciudadana & & Modos de participación \\
\hline $\begin{array}{l}\text { Evaluación de la } \\
\text { investigación }\end{array}$ & $\begin{array}{l}\text { ¿Cómo pretende } \\
\text { la organización } \\
\text { financiadora }\end{array}$ & $\begin{array}{lr}\text { ¿Cómo } & \text { se } \\
\text { seleccionan } & \text { los } \\
\text { revisores? } & \\
\end{array}$ & Criterios & $\mathrm{H}$-index, etc \\
\hline
\end{tabular}




\begin{tabular}{|c|c|c|c|c|c|}
\hline & $\begin{array}{l}\text { garantizar } \\
\text { proceso } \\
\text { evaluación } \\
\text { responsable? }\end{array}$ & $\begin{array}{l}\text { ¿Cómo } \\
\text { componen } \\
\text { paneles } \\
\text { evaluación? }\end{array}$ & $\begin{array}{l}\text { se } \\
\text { los } \\
\text { de }\end{array}$ & Membresía & $\begin{array}{l}\text { académico contra no-académico; } \\
\text { interno contra externo;ciudadanos; } \\
\text { género; disciplina; etc. } \\
\text { Funciones y proceso de toma de } \\
\text { decisiones }\end{array}$ \\
\hline & & $\begin{array}{l}\text { ¿Cómo } \\
\text { promueve } \\
\text { proceso } \\
\text { responsable? }\end{array}$ & $\begin{array}{l}\text { se } \\
\text { un }\end{array}$ & Formación, guías & $\begin{array}{l}\text { Cómo valorar contribución social, } \\
\text { Sesgo } \\
\text { transdisciplinario/interdisciplinario, } \\
\text { Concienciación de la carrera de } \\
\text { género, etc. }\end{array}$ \\
\hline & & $\begin{array}{l}\text { ¿cómo se mitiga } \\
\text { los sesgos c } \\
\text { género en } \\
\text { proceso } \\
\text { evaluación? }\end{array}$ & $\begin{array}{l}\text { de } \\
\text { de } \\
\text { el } \\
\text { de }\end{array}$ & $\begin{array}{l}\text { Mecanismos } \\
\text { institucionales }\end{array}$ & $\begin{array}{l}\text { Paneles de evaluación con equilibrio } \\
\text { de género; } \\
\text { Evaluación periódica en función del } \\
\text { género de las subvenciones } \\
\text { concedidas; } \\
\text { Consideraciones sobre la carrera } \\
\text { profesional en función del género, } \\
\text { etc }\end{array}$ \\
\hline & $\begin{array}{l}\text { ¿Cómo se apoya la } \\
\text { investigación } \\
\text { responsable en el } \\
\text { proceso de }\end{array}$ & $\begin{array}{l}\text { Historial } \\
\text { personal } \\
\text { investigador }\end{array}$ & & $\begin{array}{l}\text { Los resultados de la } \\
\text { investigación } \\
\text { (amplia gama más allá } \\
\text { de los documentos y } \\
\text { las citaciones) } \\
\end{array}$ & $\begin{array}{l}\text { Documentos } \\
\text { conjuntos de datos } \\
\text { software } \\
\text { directrices } \\
\text { etc } \\
\end{array}$ \\
\hline & evaluación? & & & $\begin{array}{l}\text { Contribución/impacto } \\
\text { social }\end{array}$ & $\begin{array}{l}\text { Narración de la contribución de la } \\
\text { sociedad } \\
\text { Declaraciones de impacto; } \\
\text { testimonios de las partes } \\
\text { interesadas; } \\
\text { Cartas de apoyo; } \\
\text { etc. }\end{array}$ \\
\hline & & & & $\begin{array}{l}\text { Medidas de } \\
\text { excelencia/calidad }\end{array}$ & $\begin{array}{l}\text { cuantitativo } \\
\text { cualitativo } \\
\text { mixto }\end{array}$ \\
\hline & & $\begin{array}{l}\text { Propuestas } \\
\text { proyectos }\end{array}$ & & $\begin{array}{l}\text { Elementos del diseño } \\
\text { de investigación }\end{array}$ & 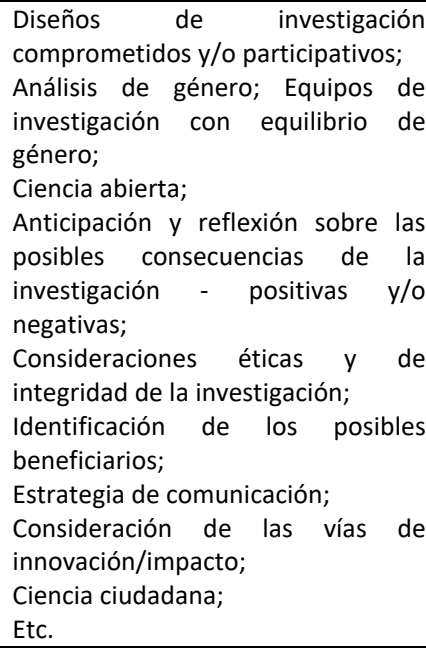 \\
\hline Monitorización & $\begin{array}{l}\text { ¿Cómo supervisa o } \\
\text { evalúa la RFO su } \\
\text { enfoque de apoyo } \\
\text { a la } \\
\text { responsabilidad? }\end{array}$ & & & $\begin{array}{l}\text { Medidas } \\
\text { procesos }\end{array}$ & $\begin{array}{l}\text { Criterios de evaluación; } \\
\text { Participación interna versus externa; } \\
\text { autoevaluación continua frente a } \\
\text { autoevaluación periódica; } \\
\text { enfoques cualitativos; indicadores }\end{array}$ \\
\hline
\end{tabular}

Fuente: Protocol for the Country Correspondent Network, Study of Research Funding Organisations (CCNRFO), V1 16 November 2020 SUPER_MoRRI project. Traducción de la autora 
Por último, se presenta la relación de los materiales analizados.

Tabla 3. Documentación del caso español

\section{Documentos}

- Ley para la Ciencia, la Tecnología y la Innovación (14/2011)

- Estrategia Española de Ciencia y Tecnología 2013-2020

- Plan Estatal de Investigación Científica y Técnica y de Innovación período 2013-2016

- Plan Estatal de Investigación Científica y Técnica y de Innovación período 2017-2020

- Programa de Actuación Anual 2020

- Programa de Actuación Anual 2019

- Programa de Actuación Anual 2013

- Plan de Acción Anual de la Agencia Estatal de Investigación para el año 2020

- Plan de Choque para la Ciencia y la Innovación 2020: hacia una economía basada en el conocimiento

- Estrategia Española de Ciencia Tecnología e Innovación 20121-2027 (Septiembre 2020)

- Informe sobre la Estrategia Española de Ciencia Tecnología e Innovación realizado por la Confederación Española de Sociedades Científicas (COSCE, Junio 2020)

\section{Web}

https://www.ciencia.gob.es/ (acceso julio-diciembre 2020)

https://www.fecyt.es/ (acceso julio 2020)

Fuente: elaboración propia

Tabla 4. Documentación europea marco

Documentos
- Commission welcomes political agreement on Horizon Europe, the next EU research
and innovation programme Brussels, 10 December 2020
- $\quad \begin{aligned} & \text { Proposal for a decision of the European Parliament and of the Council on establishing } \\ & \text { the specific programme implementing Horizon Europe - the Framework Programme } \\ & \text { for Research and Innovation Brussels, 7.6.2018 COM(2018) } 436 \text { final 2018/0225(COD) }\end{aligned}$
Web
https://ec.europa.eu/info/horizon-europe_en (acceso octubre 2019 y julio 2020).

Fuente: elaboración propia 


\section{Resultados del análisis}

\subsection{Antecedentes}

Desde el año 2013 la gobernanza del sistema se adapta a los procedimientos de management público procedentes fundamentalmente de las exigencias europeas y las distintas prioridades, objetivos y estructuras de financiación se incluyen en documentos que guían la actuación pública: a) las Estrategias Españolas de Ciencia y Tecnología que definen objetivos generales y el sentido y ordenación general del sistema. b) De la estrategia derivan los Planes Estatales que concretan dichos objetivos y sus instrumentos en los períodos 2013-2016 y 2017-2020, en el caso de la primera Estrategia 2013-2020, estando pendientes los planes de la segunda Estrategia 2021-2027. A su vez, los planes estatales y la estrategia se implementan a través de planes de actuación anuales (8 planes de actuación hasta año 2020), que fundamentalmente describen los diferentes programas de intervención y la inversión pública adscrita a cada uno de ellos, planificando y calendarizando las diversas convocatorias de fondos.

\subsection{El sistema de gobierno y gobernanza de la Ciencia y la Innovación}

Desde 2013 a 2020 el gobierno español ha estado formado por diferentes colores políticos. Desde 2011 gobernó el Partido Popular, ubicado en la derecha, mientras desde 2018, tras año y medio de sucesivas elecciones, la presidencia del gobierno fue consolidada en el por Partido Socialista Obrero Español (PSOE) en 2020, en coalición con el partido Unidas Podemos, situado más a su izquierda. Teniendo en cuenta los períodos electorales y los períodos de realización de la Estrategia Española y sus planes, ha correspondido a los gobiernos situados en la derecha su diseño y elaboración. La posibilidad de cambios en profundidad a partir de 2020 es alta dado el giro político, así como dado el impacto de la pandemia covid-19, si bien debe tenerse en cuenta en el caso español el impacto de las políticas y orientaciones europeas.

El Sistema Español de Ciencia, Tecnología e Innovación se caracteriza fuertemente por un marco de gobernanza multinivel, así como se observa la impronta de los procesos de europeización, canalizados mayormente a través de los fondos estructurales y de cohesión. Los documentos oficiales analizados explicitan claramente estos aspectos en su propia descripción del sistema. Se cita a continuación el resumen del sistema español incluido en el último Plan Estatal (20172020):

"El Sistema Español de Ciencia, Tecnología e Innovación es un sistema complejo que se caracteriza por la coexistencia de distintos sistemas regionales de investigación e innovación, con niveles de desarrollo científico y tecnológico dispares, junto con el propio sistema estatal, integrado fundamentalmente por los organismos públicos de investigación recogidos en el artículo 47 de la Ley de la Ciencia, la Tecnología y la Innovación, así como por otros organismos de investigación de ámbito estatal.

En este "sistema de sistemas», según se señala en el artículo 149.1 .15 de la Constitución Española, el Estado tiene competencia exclusiva en materia de fomento y coordinación general de la investigación científica y técnica4, mientras que la promoción y financiación de la $1+D+i$ es una responsabilidad compartida entre la Administración General del Estado y las Comunidades Autónomas5. Las diferencias regionales que presenta este "sistema de sistemas» en materia de investigación e innovación requieren políticas de fomento y generación de capacidades territoriales específicas. Los Fondos Estructurales y de Inversión Europeos para el período 20142020 incluyen entre sus objetivos temáticos la generación de capacidades de innovación para facilitar la cohesión y convergencia territoriales así como la creación de empleo y actividad económica. El marco estratégico y los objetivos específicos que permiten la aplicación de los Fondos Estructurales y de Inversión Europeos destinados a investigación e innovación lo forman 
las Estrategias de Investigación para la Especialización Inteligente (RIS3 por denominación en inglés) de las Comunidades Autónomas y la ESTRATEGIA ESPAÑOLA DE CIENCIA, TECNOLOGÍA E INNOVACIÓN 2013-2020 que, junto con los PLANES ESTATALES DE INVESTIGACIÓN CIENTÍFICA Y TÉCNICA Y DE INNOVACIÓN 2013-2016 Y 2017-2020 y sUS PROGRAMAS DE ACTUACIÓN ANUALES, constituyen la Estrategia Española para la Especialización Inteligente."

El gobierno de la política científica y de innovación corresponde por lo tanto al Ministerio de Economía, Competitividad hasta el año 2018, donde es sustituido por el Ministerio de Ciencia, Universidades e Innovación, Ciencia e Innovación desde 2020. Bajo su mandato, el sistema se forma por los siguientes organismos e instrumentos ${ }^{4}$ :

- El Consejo de Política Científica, Tecnológica y de Innovación: formado por representantes del Gobierno procedentes de diversos ministerios-ya que la política de innovación especialmente atañe a prácticamente a todos-, y las Comunidades Autónomas. Existe desde el año 2012. No hay establecida una periodicidad de las reuniones, y a fecha de diciembre de 2020 la periodicidad de las reuniones ha sido menor que anual.

- Consejo Asesor de Ciencia, Tecnología e Innovación: formado por representantes de las asociaciones empresariales y sindicatos mayoritarios, así como por representantes de la comunidad científica. Además del asesoramiento general, cuenta con la función específica de promover la introducción de mecanismos de evaluación en el sistema español.

- Sistema de Información sobre Ciencia, Tecnología e Innovación: sin ser un organismo formado por personas físicas, se cita de forma relevante este sistema que "es el instrumento de captación de datos y análisis para la elaboración y seguimiento de la Estrategia Española para la Ciencia, Tecnología e Innovación y de los Planes Estatales de Investigación, Desarrollo e Innovación" ${ }^{\prime 5}$. Dado su carácter, será tratado más adelante cuando se especifiquen los contenidos en términos de monitorización del sistema español.

Estos organismos e instrumentos tienen como finalidad asesorar e informar sobre la estrategia y planes. Además, para el seguimiento del último plan se observa una estructura específica de gobernanza (Plan Estatal 2017-20), organizada en tres niveles: dirección, coordinación y seguimiento. La dirección está a cargo de la Comisión Delegada del Gobierno en Ciencia, Tecnología e Innovación y en el Comité de Dirección del Plan Estatal, liderado por la Secretaría de Estado de Investigación, Desarrollo e Innovación. Este comité debe reunirse una vez al año contando con representantes de áreas de la Administración General del Estado (AGE) que estén involucradas en acciones de ciencia o innovación (promoción o financiación). De este comité dependen a su vez dos subcomités: a) comité de coordinación del Plan con los siguientes epígrafes: coordinación AGE y coordinación de las Acciones Estratégicas incluidas en el plan (Salud, Economía y Sociedad Digital, Industria Conectada 4.0). Aunque no es visible en el gráfico que presenta esta estructura, en el texto que la acompaña se cita que la coordinación del Plan incluirá una atención a los organismos de la AGE a los que atañe el impacto de las actuaciones incluidas en el Programa Estatal de I+D+l orientada a los Retos de la Sociedad. Por su parte el subcomité de seguimiento se dedicará a tareas que son en cierto modo ejecutivas, como contribuir a la elaboración de los Programas de Actuación Anuales, la elaboración de la

\footnotetext{
4 Así se define en la propia web del Ministerio https://www.ciencia.gob.es/portal/site/MICINN/menuitem.8ce192e94ba842bea3bc811001432ea0/?vg nextoid=8e5db1c445673410VgnVCM1000001d04140aRCRD (consulta 11 de julio de 2020).

5 ibid
} 
propuesta de informe anual de seguimiento del plan, informar de las modificaciones en las convocatorias y requisitos de elegibilidad, informar sobre las ejecuciones presupuestarias y sus modificaciones, así como tiene un papel fundamental en el sistema de datos que alimenta la gobernanza de las políticas:

"f) identificar, definir e informar de la evolución de los indicadores de gestión, seguimiento y resultados de cada una de las actuaciones recogidas en los Programas Anuales de Actuación así como los indicadores de seguimiento y evaluación del Plan Estatal; (g) contribuir al desarrollo del Sistema de Información de Ciencia, Tecnología e Innovación (SICTI) participando en el grupo de trabajo designado para tal fin, y (h) elaborar la propuesta del informe anual de seguimiento del Plan Estatal." El seguimiento está establecido cada dos meses; respecto a sus actores participantes, el liderazgo de esta subcomisión corresponde a la Dirección General de Política de Investigación Desarrollo e Innovación y participan representantes de otros Ministerios, así como de las agencias de financiación responsables del Plan. Dichas agencias son la Agencia Estatal de Investigación (AEI) y el Centro para el Desarrollo Tecnológico e Industrial (CDTI), descritas a continuación.

La AEI fue creada en el año 2015 y se presenta en la web del Ministerio como un "nuevo modelo de gestión", destinado a mejorar la planificación y "dotar a las convocatorias de la estabilidad que requieren las actividades de investigación". Fundamentalmente gestiona la financiación pública destinada a investigación. Se indica igualmente que su finalidad es garantizar "la rendición de cuentas", "racionalizar" o "simplificar y estandarizar los procedimientos". Su gobierno se canaliza a través de un Consejo Rector presidido por el Ministro, una Comisión de Control y un Comité Científico-Técnico nombrado en 2017 en el que participan 12 miembros relevantes de la ciencia española. Dos de ellos se relacionan con las Ciencias Sociales y Humanidades (un economista y una historiadora). Ninguno de los participantes es experto 0 experta en política científica o de innovación. ${ }^{6}$

Por su parte, CDTI es una Entidad Pública Empresarial dependiente del Ministerio de Ciencia e Innovación y canaliza el apoyo económico público a empresas. Se declara que su objetivo es "contribuir a la mejora del nivel tecnológico de las empresas españolas". También cuenta con oficinas representantes en países como Bélgica, Japón o Brasil para apoyar la internacionalización de estas actividades y la consecución se proyectos tecnológicos de gran calado como los vinculados a la Agencia Europea del Espacio, así como apoya a las empresas que innovan en sus desgravaciones fiscales. Como empresa pública cuenta con un Consejo de Administración presidido por la Secretaria General de Innovación del Ministerio de Ciencia e Innovación.

Teniendo en cuenta los objetivos de racionalización citados, cabe incluir en este sistema a los órganos encargados de la evaluación: la Agencia Nacional de Evaluación de la Calidad y Acreditación, constituida en 2014 a través de la Ley de Racionalización del Sector Público (Ley15/2014 de 16 de Septiembre) que cuenta como objetivo "contribuir a la mejora de la calidad del sistema de educación superior mediante la evaluación, certificación y acreditación de enseñanzas, profesorado e instituciones".

Por su parte la Comisión Nacional Evaluadora de la Actividad Investigadora (CENEAI) se encarga de la evaluación investigadora para el Consejo Superior de Investigaciones Científicas (CSIC), el mayor órgano de investigación del país.

6

https://www.ciencia.gob.es/portal/site/MICINN/menuitem.8ce192e94ba842bea3bc811001432ea0/?vg nextoid=2e96168d238aa510VgnVCM1000001d04140aRCRD, visitado el 7 de agosto de 2020. 


\subsection{Definición de prioridades y participación pública}

La definición de las prioridades de financiación se articuló en España en el período 2013-2020 a través de la citada Estrategia Española de Ciencia y Tecnología y de Innovación 2013-2020 realizada con la participación de agentes públicos, económicos y sociales (sindicatos), así como actores del sistema de ciencia e I+D y una consulta pública que recibió 1.400 comentarios.

Por su parte, el Plan Estatal 2013-2016 fue realizado según se incluye en el texto del propio plan, con la siguiente participación: distintas unidades de la Administración, expertos de la comunidad científica, técnica y empresarial, incluyendo participación internacional, así como asociaciones empresariales y sindicatos, representantes de los centros públicos de investigación y las universidades, centros tecnológicos y unidades de transferencia o diversas plataformas tecnológicas. Fue sometido a consulta pública (noviembre 2012) y recibió más de 800 comentarios. Se cita explícitamente que estos comentarios fueron debidamente analizados e incorporados en la medida de lo posible. Se cita que las Comunidades Autónomas fueron informadas a través de la comisión correspondiente del Consejo de Política Científica y Tecnológica, donde participan de forma directa.

Finalmente, para la elaboración del Plan 2017-2020 se cita que se contó con: "setenta expertos independientes que incluyen a: (a) responsables de la programación y gestión de ayudas de $1+D+i$ y responsables de las políticas sectoriales de la Administración General del Estado; (b) investigadores de universidades, OPIs, organismos de investigación, empresas, centros tecnológicos, plataformas tecnológicas, centros de investigación del Sistema Nacional de Salud, etc.; (c) representantes de la Conferencia de Rectores de las Universidades Españolas, las sociedades científicas, asociaciones empresariales, plataformas tecnológicas, asociaciones de usuarios de ayudas del Plan Estatal, etc., y (d) otros expertos con una experiencia contrastada en el diseño de políticas públicas de $1+D+i$ a nivel nacional e internacional." También fue sometido a consulta pública (el resultado fueron 170 documentos con comentarios) y el Consejo Asesor antes citado emitió también su juicio.

En septiembre del año 2020 aparece la nueva Estrategia Española de Ciencia, Tecnología e Innovación 201-2027. La participación de diversos actores, incluidos aquellos públicos o administrativos está mucho más detallada en la estrategia, observándose un giro con respecto a los documentos precedentes. En concreto se cita que "La elaboración de la EECTI ha seguido un proceso abierto e inclusivo de los principales agentes del SECTI, no solo de "arriba abajo" a través de la propia gobernanza establecida en la Ley 14/2011,

de 1 de junio, de la Ciencia, la Tecnología y la Innovación (LCTI) sino, además, "de abajo arriba" a través de la constitución de grupos de interés que responden a un modelo de cuádruple hélice (Empresas, Investigación y Administración Pública, Sociedad Civil y Usuarios de Innovación)." Además de los actores clásicos citados anteriormente como los Consejos, se detalla que se formó un Grupo Asesor contando con representantes del Ministerio, de las agencias estatales (AEI, CDTI, Instituto Salud Carlos III) y FECYT, así como representantes de instituciones europeas de ciencia e innovación y fondos europeos. El informe cita la consulta a diversas sociedades científicas externas al sistema como la Confederación de Sociedades Científicas de España o la Conferencia de Rectores. Sin embargo, no queda clara cuál ha sido la participación de la sociedad civil, esa pata de la Cuádruple Hèlix, no se detallan las organizaciones participantes. 


\subsubsection{La Estrategia Española de Ciencia y Tecnología e Innovación 2013-2020}

Dicha estrategia se concibió en un momento de grave crisis económica en el país. Su marco estaba claramente enfocado en el aumento la competitividad en un contexto de austeridad de recursos. Se basa en un diagnóstico en el que se evalúa el nivel científico como adecuado, mientras que la principal debilidad radica en la falta de innovación empresarial. El objetivo es estimular el progreso social y económico a través de economías basadas en la innovación. La idea fundacional destacada es la siguiente: "desde la idea al mercado". Con esta Dirección prioritaria, la estrategia estableció cuatro objetivos: promoción del talento y su empleabilidad, generación de conocimiento y fortalecimiento del sistema, liderazgo empresarial en I+D+i e I+D+i orientado a los retos de la sociedad.

La visión que se ofrece de la ciencia, la innovación y la tecnología es claramente positiva. En ningún caso hay comentarios sobre los posibles riesgos o consecuencias no deseadas que deberían ser mitigadas por las políticas nacionales, regionales 0 institucionales. La responsabilidad y sus aspectos asociados están escasamente presentes y no están relacionados con los planteamientos sobre la excelencia. La excelencia está vinculada a la competitividad y a la extensión de las fronteras del conocimiento. Las políticas de transferencia están orientadas al mercado y a la propiedad intelectual.

Se observan los siguientes aspectos relacionados con la RRI, aunque su enfoque no se corresponde con muchos de los marcos habituales de la RRI, que suelen ser críticos y están orientados al cambio sistémico. Dado lo sucinto de las menciones observadas en esta estrategia, se incluye un resumen a modo de guión a continuación:

- Participación pública: la estrategia ha sido elaborada por los agentes tradicionales del sistema de Ciencia e Innovación (interesados en la ciencia y la innovación, agentes públicos, empresas, sindicatos), y fue objeto de una consulta pública. No parecen estar presentes las concepciones vinculadas a la RRI como sistemas de innovación centrados en la cuádruple o quíntuple hélice, en los que los agentes sociales no tradicionales (otras asociaciones civiles además de los sindicatos) participan en la definición de las políticas; no se hallan menciones. No obstante, se citan las estrategias regionales de especialización inteligente como lugar de participación pública "más amplia".

- Los desafíos sociales (salud, sostenibilidad, suministro de energía, otros) se citan como objetivos de la investigación. Estos desafíos se derivan de los desafíos mundiales y de las estrategias europeas (Estrategia 2020). Al ser concebidos como objetos de investigación, no parecen implicar la participación pública o las estrategias de gobernanza en su desarrollo futuro (no se citan).

- Inclusión social: también es un objetivo de investigación dentro de los desafíos sociales mencionados. No se hace referencia a las políticas de inclusión en las propias instituciones científicas o tecnológicas.

- Igualdad de género: se hacen menciones generales, en línea con el marco europeo del momento, con especial referencia a la participación femenina en la Ciencia e Innovación.

- Acceso abierto: se ha enmarcado dentro de los principios de gestión, como un instrumento que puede favorecer la colaboración y corresponsabilidad de los agentes.

- Educación científica: enmarcada en el fomento de la cultura científica y empresarial, relacionada con la divulgación, la promoción de las carreras científicas y el fomento de 
percepciones positivas sobre la ciencia y la tecnología. No se hace mención de posibles iniciativas de co-creación o de ciencia ciudadana.

- Responsabilidad social de las empresas: enmarcada en la promoción de la colaboración público-privada en materia de inversión en ciencia e innovación (patrocinio empresarial), así como en la consideración de la innovación como parte de la responsabilidad del progreso social.

Otros aspectos relacionados con la RRI, como la ética, la reflexividad o los mecanismos de respuesta, no se citan en la estrategia; no se utilizan estos términos específicos ni se refieren a conceptos de significado cercano.

\subsubsection{Planes 2013-2020}

Como se citaba, la Estrategia Española de Ciencia y Tecnología y de Innovación 2013-2020 define grandes objetivos y alcance de los ámbitos de intervención. Corresponde a los Planes Estatales de Ciencia y Tecnología y de Innovación el desarrollo y financiación de lo planteado en la Estrategia.

El Plan Estatal De Investigación Científica y Técnica y De Innovación para el Período 2013-2016 sigue en gran medida la línea marcada en la Estrategia, ya que ha sido desarrollado en un contexto muy parecido, donde la situación económica es grave y el sistema político español está marcado por casos crecientes de corrupción y baja confianza en las instituciones. El Plan destaca que "Por tanto, la asignación de fondos públicos se otorgará por procedimientos de concurrencia competitiva y la selección de las propuestas financiadas se realizará teniendo en cuenta criterios científico-técnicos y, en su caso, criterios de viabilidad tecnológica, empresarial y comercial amparados". La elaboración de este plan coincidió en gran medida con el debate a nivel europeo del programa marco Horizonte 2020, si bien su impronta en cuanto a RRI no es perceptible en este Plan. Es clara en cambio en el siguiente, como se detallará en breves.

Los diez objetivos del plan 13-16 se dirigen a mejorar la calidad, el liderazgo e internacionalización, la inversión e implicación del sector privado y la colaboración públicoprivada, mientras el último objetivo es "Estimular la $I+D+l$ orientada para respuesta a los retos de nuestra sociedad". Otro de los objetivos se dirige a estimular la cultura científica y de innovación, si bien en un plano unidireccional en el que no se cita la participación del público, sino actividades enmarcadas fundamentalmente en la comunicación y la divulgación desde el ámbito científico a la sociedad. El Plan introduce un gran cambio estructural de la financiación, que ya no se dirige a sectores o disciplinas, sino a problemas o retos a los que es necesario dar respuesta, lo cual podría favorecer en términos de gobernanza a la RRI y sus aspectos asociados. Estos retos se corresponden con los objetivos definidos en la estrategia y dan lugar a cuatro programas estatales donde se estructura la financiación. Estos programas, con ligeras modificaciones, siguen vigentes en el siguiente Plan y por tanto en 2020. Los programas se dirigen a las siguientes temáticas: promoción e incorporación del talento, fomento de la excelencia en la investigación científica y técnica, liderazgo empresarial en $1+D+\mid$ e $1+D+1$ orientada a los retos de la sociedad.

La definición de los retos sociales pretende alinearse explícitamente con lo definido en H2020 europeo e incluye como elementos transversales la investigación en Ciencias Sociales e Humanidades, así como la perspectiva de género. Sin embargo, no se detallan cuestiones en torno a estos aspectos, y los retos sociales tienen un marcado interés de mercado y productividad. Los retos sociales definidos están relacionados con la salud, la emergencia climática y la sostenibilidad, el transporte integrado, la digitalización, la energía, la protección y defensa así como los cambios e innovaciones sociales. La presencia de aspectos RRI en los retos es escasa, si bien se podrían observar marcos favorables para la misma como los siguientes: el 
foco en las personas frente a la enfermedad citado como objetivo en el reto de salud, o las innovaciones sociales como elemento crítico en términos de salud y demografía. Las innovaciones sociales o referencias similares son sin embargo escasamente citadas en los retos de forma global (defensa, transporte o energía), primando un enfoque fundamentalmente técnico de los cambios que finalmente conducirán a solucionar los retos, aunque hayan sido definidos dentro de un programa denominado retos de la sociedad. Por su parte el reto específico relacionado con los cambios e innovaciones sociales se dirige a aspectos como el desafío migratorio o la desigualdad, y, en primer lugar, al desarrollo económico y creación de empleo, el diseño y la evaluación de las políticas públicas, la protección del patrimonio así como a la "innovación, cambio técnico, progreso y bienestar" en el que se financiarán aspectos relacionados con nuevos modelos organizativos, la internacionalización de las actividades económicas, modelos de liderazgo empresarial y "comportamientos y aceptación social de la tecnología, la innovación y el riesgo". En este último punto no se detalla más, por lo que es difícil saber si el impacto indeseado de la ciencia o la innovación podría contarse entre sus orientaciones.

De forma global, las menciones transversales a lo social son escasas en los distintos programas, mientras que se observa que, en los objetivos relacionados con lo social, la presencia del imperativo de desarrollo económico y sus aspectos asociados están muy presentes.

Es difícil en cualquier caso saber hasta qué punto este tipo de líneas encaminadas a lo social han estado presentes en la financiación final de proyectos, ya que no se especifican como criterio de elegilibidad en el Plan que primero aterriza la estrategia, si bien algunos aspectos pueden haber sido incorporados a las convocatorias de fondos, como lo relacionado con la presencia femenina. Sin embargo, aunque no se hayan definido como principios generales, sí se cita en relación a las actividades de la Agencia Estatal de Investigación-encargada de los fondos de investigación- que "asignará los recursos públicos utilizando criterios internacionalmente validados en relación a los méritos científicos o técnicos de las propuestas y tendrá en cuenta el impacto socioeconómico potencial de las mismas", mientras el CDTI-a cargo de los fondos de innovación- los asignará en relación al "mérito tecnológico, la novedad y oportunidad, aplicabilidad y viabilidad industrial, la proximidad del mercado y el impacto socioeconómico de los resultados". Parece, por tanto, recaer la responsabilidad en estas agencias de la inclusión de criterios que puedan estar relacionados con la RRI.

El plan 2013-2016 define así mismo, principios de gestión y buen gobierno, si bien aspectos éticos o relacionados con la RRI no están presentes, estando orientados a la eficiencia (integración de fondos, facilitación de la base "desde la idea al mercado", etc). De forma global puede decirse que el este Plan no desarrolla aspectos de RRI citados en la estrategia.

Por su parte el Plan Estatal 2017-2010, ubica al Plan 2013-2016 como un elemento que "introdujo una visión integrada de la investigación, el desarrollo tecnológico y la innovación, superando la idea de que la I+D+i son fases secuenciales de un proceso lineal." Este nuevo plan mantiene la misma estructura y los mismos programas estatales, si bien sí se observa la impronta de la RRI en su misma definición de objetivos. Entre sus ocho objetivos, además del referido a los retos sociales, al igual que en el plan anterior, se observa el siguiente: "Promover un modelo de I+d+i abierto y responsable apoyado en la participación de la sociedad".

En este objetivo se citan aspectos como la interdisciplinariedad, la ciencia abierta, la ética en la investigación, la dimensión de género no sólo referida a la presencia de mujeres, sino también a la dimensión de género en los contenidos de ciencia e innovación, el impulso de una agenda ciudadana en la configuración de los retos científicos y de innovación a desarrollar, el estímulo de las vocaciones científicas, así como la inclusión de las personas con discapacidad. En relación a este último punto, cabe destacarse la gran tradición social española en este campo, 
desarrollada con gran presencia desde los años ochenta, en comparación con otros aspectos relacionados a la inclusión y no discriminación. Destaca en la definición de este objetivo la citación explícita de "El desarrollo y usos de nuevas aplicaciones y tecnologías de minería y análisis de textos y datos que permitan desarrollar nuevos indicadores de impacto de las políticas de I+D+i."

Así mismo, los principios han cambiado: "La asignación de fondos públicos a través de los Planes Estatales se otorgará de acuerdo a los principios de publicidad, transparencia, concurrencia, objetividad y no discriminación así como de eficacia en el cumplimiento de los objetivos fijados por la Administración otorgante y eficiencia en la asignación y utilización de los recursos públicos [...]", siendo no discriminación una novedad.

Este nuevo plan se basa en una evaluación del anterior, en el que destacan algunos retos (R) y oportunidades-relacionados o inhibidos en cuanto a RRI-en sus 4 objetivos generales, que siguen siendo aquellos definidos en la Estrategia 13-20.

- En materia de investigación e innovación empresariales no hay retos ni oportunidades vinculados a RRI, dado lo explícito interés en el reto definido como sigue: "Marco regulatorio favorable a la innovación y desarrollo tecnológico "

- En materia de recursos humanos se hace referencia, como oportunidad, al fomento y la incorporación de carreras de mujeres, mientras la retención y atracción del talento son definidas como un reto. La crisis económica ha limitado el número de investigadores presentes en el país, así como su formación e incorporación.

- En cuanto a I+D+l y los retos de la sociedad se cita como oportunidad la Investigación y la innovación abiertas, con una creciente participación ciudadana (R2)

- En relación a la generación de conocimientos e infraestructuras de investigación: se ubican como retos los siguientes aspectos: Impacto científico y social (R2), Fortalecimiento y acceso a ICTs, interoperabilidad y modernización de infraestructuras y equipación (R3), Redes de colaboración científica e integración transdisciplinar (R4), Difusion de los resultados a la sociedad (R5), Acceso Abierto y Ciencia Abierta (R5).

Se observa por tanto que lo relacionado con RRI parece situarse mayoritariamente en un plano de reto que de oportunidad, si bien no de forma unívoca.

El plan establece principios generales que lo guían: eficiencia y eficacia (incluyendo la estandarización de los procedimientos de solicitud, evaluación y justificación), transparencia y rendición de cuentas (incluye acceso abierto) y ética de la investigación, donde se citan aspectos como "una investigación con para la sociedad", el incremento de la confianza social en la ciencia, así como que la observación de este principio en las ayudas concedidas será responsabilidad del Comité Español de Ética de la Investigación establecido en la citada Ley 2011, e integrado por representantes de la comunidad científica y tecnológica.

Así mismo, en el programa estatal relacionado con los retos de la sociedad se observa que, además de referencias al aumento de la competitividad y a acelerar el proceso del "laboratorio al mercado", en este plan hay un mayor énfasis en la participación: "El impulso de iniciativas de ciencia ciudadana en temas en los cuales los cambios y el impacto de la l+D y de la innovación están asociados estrechamente a una mayor participación y grado de compromiso de la sociedad." Así mismo se observa la aparición de términos cercanos a aproximaciones más responsables en otros retos incluidos en el programa de retos de la sociedad (energía, transporte, defensa, etc) que no estaban en el anterior Plan, como los siguientes: investigación 
traslacional, bioeconomía, estrategias económicas circulares, eco-innovación y eco-diseño. También se observa el fomento de la ciencia ciudadana como un aspecto relevante en relación al cambio climático. Por último, el reto anteriormente definido como "Cambios e innovaciones sociales" se ha rebautizado como "Ciencias Sociales y Humanidades y Ciencia con y para la Sociedad", y se plantea como una investigación fundamental para el resto de retos (energía, transporte, economía digital etc).

En este Plan hay más referencias explícitas a RRI, mientras se observa que el marco general es igualmente una visión netamente positiva de la innovación y la tecnología. Ningún reto específico relativo a una mala utilización de la tecnología aparece mencionado. Como ejemplo, se citan la digitalización e incremento de los sistemas de captación masiva de datos y vigilancia, sin hacer referencia a la privacidad y su protección, aunque la merma de este aspecto y la necesidad de plantear soluciones sea altamente debatido y mediático.

Por su parte, las referencias a la responsabilidad social corporativa (RSC) del ámbito empresarial siguen enmarcadas en la responsabilidad del sector privado como financiadores de la innovación y su rol de mecenazgo, sin dirigirse a la labor interna de las empresas en materia laboral, de igualdad $u$ otros aspectos incluidos de forma habitual en el marco RSC.

De forma global, este último plan parece incorporar el lenguaje y estructura de lo dispuesto por las instituciones europeas en materia de RRI. Los documentos analizados adaptan o dotan de contenido específico para el contexto español de forma limitada y poco apreciable.

\subsubsection{La Estrategia Española de Ciencia, Tecnología e Innovación 2021-2027.}

En septiembre del año 2020 aparece la nueva Estrategia Española de Ciencia, Tecnología e Innovación 2021-2027. El color político del gobierno ha cambiado hacia la izquierda con respecto a las estrategias y planes precedentes. El documento refleja las adaptaciones de la RRI en la nueva programación europea Horizonte Europa, que como se destacaba en la introducción, incide especialmente en los siguientes aspectos: la co-creación y la ciencia abierta.

Previamente, en junio de 2020 la Confederación de Asociaciones Científicas de España emite un informe con respecto al primer borrador. Sus principales demandas son una mayor alineación con las políticas europeas, que a su entender, destilan el siguiente principio: "sitúa precisamente a la política científica en el centro de la política, y es el punto de apoyo para que en España, en línea con Europa, la ciencia sea considerada un elemento transversal de las políticas públicas." Esta es la mayor reclamación, conjuntamente con la mejora del sistema de gobernanza y de indicadores, al que se reclama incorporación de nuevas técnicas como Big Data o Al en el marco de la digitalización general de la captura de datos, así como indicadores de impacto y no sólo de output o resultados. A su vez demanda "especificar la relación entre objetivos que se pretende cumplir e indicadores para, de esta manera, poder hacer un seguimiento del cumplimiento de la estrategia." Por último, indica que la principal función de la evaluación no debe ser distribuir fondos, sino también la toma de decisiones estratégicas y se señala como referentes el sistema británico o canadiense. Así mismo se señala la necesidad de cambiar el sistema de gobernanza para cuestiones tales como "establecer medidas diferenciadoras que mantuvieran vinculados al sistema aquellos investigadores más activos y, a la vez, propiciaran la salida de los más ineficientes". Por otro lado, el informe critica el diagnóstico y enfoque general de la estrategia en el siguiente sentido: "Del documento parece desprenderse que el gran problema de España no es la generación de ciencia de excelencia, sino su transferencia a la empresa privada. [...] Aunque éste es sin duda un problema de nuestro país (que no exclusivamente del sistema de $1+D+i)$, centrar toda la Estrategia en intentar resolverlo es un error. No es que las medidas propuestas no puedan ser adecuadas, es que faltan otras muchas que deberían ir destinadas a 
mejorar la generación de conocimiento como motor de la innovación en nuestro país, y a poner mayor énfasis en la componente social de la ciencia."

A este respecto también se indica que "es necesario impulsar una investigación que, además de ser capaz de ser utilizada por el tejido industrial y competitivo a corto y medio plazo, contribuya a solucionar problemas sociales. La componente social de la investigación es esencial en estos momentos. Identificar y ejecutar este tipo de acciones de forma eficaz no es una tarea trivial y debe tener como elemento referente el incremento de la calidad del conjunto de nuestro sistema."

Por último, cabe destacar la demanda de una implicación en el Plan S que varios países europeos han suscrito para impulsar la ciencia abierta. Estos planes están dirigidos a limitar el poder que algunas editoriales científicas imponen a los estados.

La Estrategia Española de Ciencia, Tecnología e Innovación 201-2027 finalmente aprobada por el Consejo de Ministros, incorpora menciones en las direcciones señaladas- con distinto grado de intensidad- exceptuando referencias a captación de datos mediante nuevas técnicas o al Plan $S$, si bien la ciencia abierta está muy presente en el documento.

Cabe destacar el contexto de elaboración de la Estrategia, comenzada en febrero de 2020 y por tanto durante el despegue de la pandemia, y finalizada en septiembre de 2020. El preámbulo de la Estrategia cita que es un momento en el que se está repensando el papel de la ciencia y la innovación en la sociedad. En el caso español, el punto de partida es un "retroceso en la $1+D+1$ durante los últimos 15 años", así como el efecto económico "devastador" de la pandemia. Esta nueva crisis ha puesto de manifiesto las debilidades del sistema de ciencia e innovación español. También "La crisis sanitaria ha puesto a la ciencia en un lugar preeminente de la sociedad española y de los gobiernos de España y de las Comunidades Autónomas (CCAA) para la toma de decisiones". En esta línea, y a diferencia de anteriores documentos se incluyen actuaciones en el Eje 2, que "fomentará el desarrollo de los instrumentos y órganos dependientes de la LCTI para incrementar el asesoramiento científico experto a los distintos estamentos estatales y de gobierno. De esta manera, la I+D+l estará presente en la toma de decisiones, contribuyendo a la resolución de situaciones de crisis en base al conocimiento." En otras partes del documento se observa que se quiere trabajar para hacer la innovación "mainstreaming": "Innovación sistémica y sistemática que permitirá que la innovación esté presente en todos los ámbitos de la sociedad, públicos y privados, y en todos los sectores, en particular, en los grandes objetivos del país, en las misiones estratégicas españolas, en las compras de la administración, etc."

Se observa por tanto un incremento acerca de las expectativas y alcance de la ciencia, así como la Estrategia cuenta con fines más ambiciosos y sociales que la anterior. El desarrollo económico basado en el conocimiento es uno de los fundamentales, pero se apela claramente a lo largo del documento a los "retos y desafíos de la sociedad", con un fin más ambicioso y cercano a los presupuestos RRI que proponen un cambio de carácter sistémico: "encaminar a España y a Europa hacia una senda de recuperación resiliente, sostenible y justa." o "progresar hacia una economía basada en una tecnología baja en carbono, digitalizada, sostenible y socialmente responsable". El crecimiento económico se acompaña de otros aspectos, mientras aparecía en solitario en anteriores documentos.

El papel de esta estrategia en el marco de gobernanza europeo es más explícito si cabe que en los anteriores documentos, donde ya estaba por otro lado muy presente. En este caso el documento debe considerarse también como la Estrategia de Especialización Inteligente de España, conjuntamente con las estrategias S3 regionales. El documento ordena por tanto el marco de recepción de fondos europeos estructurales, del programa marco de investigación, así como del fondo de recuperación que se está generando por la pandemia. 
Así mismo, la estrategia se enmarca claramente en acuerdos globales, como "Objetivos de Desarrollo Sostenible (ODS) de la Agenda 2030 de las Naciones Unidas y del Acuerdo de París". Especialmente los ODS aparecen repetidamente a lo largo del documento, como marco de referencia y siendo citada la creciente importancia que la ciencia y la innovación tienen en los propios ODS, remarcando por tanto la contribución que desde esta área se debe hacer a los mismo: "En la Agenda de Adís, con un capítulo dedicado a la Ciencia, la Tecnología y la Innovación, los ODS cobran especial relevancia al ser considerados herramientas fundamentales para mejorar la eficiencia en las dimensiones económica, social y medioambiental". La estrategia comparte las orientaciones políticas definidas por la UE, que son las siguientes, donde se observa un marco más cercano a RRI.

- el cambio demográfico,

- la globalización de la economía,

- la digitalización,

- la industria y la sociedad,

- el control del cambio climático a través de una transición ecológica justa y social.

Por su parte, en el análisis DAFO se observa que los aspectos asociados a RRI citados son género, ciencia abierta y percepción social de la ciencia. Todos se sitúan en formalezas y oportunidades. También se cita como oportunidad el "Papel clave de la ciencia y la innovación en la consecución de los ODS", si bien no a la inversa, es decir que los ODS vayan a contribuir a una mejor ciencia. En el marco de debilidades y amenazas se observan dos aspectos que no suponen el pilar del marco habitual de la RRI, pero están relacionados y apelan a problemáticas concretas del sistema español: el bajo impacto en transferencia a sistema productivo y sociedad de la ciencia española, así como la precariedad del personal investigador más joven.

De forma general, los aspectos que ya se han citado (ciencia y sociedad, género, ciencia abierta, co-creación etc) están muy presentes en la estrategia y su modelo. De los 4 principios que la guían, uno apela a la perspectiva de género y el último a: "La Responsabilidad social y económica de la I+D+l a través de la incorporación de la ciencia ciudadana y la aplicación de la co-creación $y$ las políticas de acceso abierto, así como, el alineamiento de la $1+D+l$ con los valores, necesidades y expectativas sociales". El "marco social" está incluido entre los tres elementos transversales, así como puede encontrarse entre los objetivos (el primer objetivo destaca el papel de la ciencia y la tecnología para conseguir los ODS y la Agenda 2030), ejes (ciencia e innovación en la sociedad) y resultados esperados (reconstrucción social y económica, empleo de calidad, mejora del impacto social de la ciencia y la innovación).

Por su parte en cuanto a temáticas prioritarias, las líneas estratégicas nacionales se enmarcan en los grupos temáticos de Horizonte Europa (1. Salud 2. Cultura, Creatividad y Sociedad Inclusiva 3. Seguridad Civil para la Sociedad...) En cada línea se observan temáticas de carácter más social, como ejemplo, en la línea 3 se observa que se incluye lo siguiente: Dimensión espacial de las desigualdades, migraciones y multiculturalidad y Monopolios y poder de mercado: medición, causas y consecuencias. En este caso se alude a problemas causados por el mercado, aspecto que anteriormente había sido igualmente tratado de forma netamente positiva. A continuación la relación completa de líneas estratégicas:

\begin{tabular}{|l|}
\hline 1. Salud \\
\hline - Medicina de precisión \\
\hline - Enfermedades infecciosas \\
\hline - Nuevas Técnicas diagnósticas y terapéuticas \\
\hline
\end{tabular}


- Cáncer y Gerociencia: Envejecimiento, enfermedades degenerativas

2. Cultura, Creatividad y Sociedad Inclusiva

- Evolución humana, antropología y arqueología

- Cognición, lingüística y psicología

- Filología y literaturas hispánicas

3. Seguridad Civil para la Sociedad

- Dimensión espacial de las desigualdades, migraciones y multiculturalidad

- Monopolios y poder de mercado: medición, causas y consecuencias

- Ciberseguridad

- Protección ante nuevas amenazas para la seguridad

4. Mundo digital, Industria, Espacio y Defensa

- Inteligencia Artificial y Robótica

- Fotónica y electrónica

- Modelización y análisis matemático y nuevas soluciones matemáticas para ciencia y

tecnología

- Astronomía, Astrofísica y Ciencias del Espacio

- Internet de la próxima generación

- Nuevos materiales y técnicas de fabricación

5. Clima, energía y movilidad

- Cambio climático y Descarbonización

- Movilidad sostenible

- Ciudades y ecosistemas sostenibles

6. Alimentación, Bioeconomía, Recursos Naturales y Medio Ambiente

- Exploración, análisis y prospectiva de la biodiversidad

- Cadena agroalimentaria inteligente y sostenible

Se espera que esta estructura "incentivará la multidisciplinariedad que permitirá el desarrollo de misiones científicas y el abordaje proyectos tractores de los ODS". Aparece así mismo una somera referencia a la pujante "science diplomacy" en los siguientes términos: "En el ámbito de la cooperación científica y la colaboración internacional, que incluye la cooperación internacional para el desarrollo y la diplomacia científica, se dotará a las delegaciones españolas en países prioritarios de personal especializado". En el apartado referido al aspecto transversal "Marco Social" se incluyen otras referencias de interés: a la comunicación científica inclusiva, a la sociedad como creadora de innovación social, o acciones contra las "fake news". El principal puntal es que "la sociedad civil debe ser un elemento central del SECTI[Sistema Español de Ciencia, Tecnología e Innovación]. Dicho marco conceptual está contemplado, igualmente, en las políticas de ciencia europeas y en las iniciativas a favor de la ciencia abierta."

Como se citaba anteriormente, no se ha detallado cómo ha participado la sociedad civil en la elaboración de la estrategia. Sin embargo, en el apartado dedicado a la futura gobernanza de la estrategia se menciona, entre otros aspectos que "la LCTI [Ley de Ciencia, Tecnología e Innovación], prevé los siguientes ámbitos de gobernanza del SECTI":

- La Estrategia Española de Ciencia, Tecnología e Innovación

- El Consejo de Política Científica, Tecnológica y de Innovación (CPCTI)

- El Consejo Asesor de Ciencia, Tecnología e Innovación (CACTI)

- El Comité Español de Ética de la Investigación (CEEI)

- El Sistema de Información sobre Ciencia, Tecnología e Innovación (SICTI)" 
Es interesante destacar que dos son instrumentos (estrategia y sistema de información), no actores. Otros documentos europeos se suman a la gobernanza, citándose que serán tenidos en cuenta las recomendaciones: Programa de Estabilidad de 2020 de España, así como la Guía para las Smart Specialization Strategies (S3).

De este marco (S3) se deriva el modelo de 4 Hélix que se utiliza para armar el comité de seguimiento de la Estrategia que contará con los actores habituales (CCAA, ministerio, reconocidos científicos y/o tecnólogos, AEI, CDTI, FECYT, Carlos III) a los que se suman "Dos representantes de la sociedad, dos representantes sindicales y dos representantes del ámbito empresarial que recojan las demandas de los ciudadanos, consumidores y organizaciones sin ánimo de lucro. A designar por la Secretaría General de Investigación a propuesta del CACTI".

De manera global, por tanto, lo relacionado con RRI está mucho más presente. Sin embargo, se siguen detectando dos grandes ausencias relacionadas con el marco responsable. En primer lugar, en los objetivos y actuaciones dirigidas al sector empresarial, lo social sigue sin aparecer. No se cita explícitamente, si bien se ha ampliado la visión sobre desarrollo económico.

En segundo lugar, se sigue la estela de las estrategias y planes anteriores y se muestra una visión netamente positiva de la ciencia y la tecnología, que se refuerza tras el impacto de la pandemia. No hay referencias a los posibles impactos o consecuencias negativas e indeseadas, mientras se propone un aumento de la influencia de la ciencia y la innovación en la toma de decisiones políticas, así como una intención de que la innovación sea algo transversal al resto de políticas. Se observa, no obstante, un reconocimiento a dicha problemática en el Anexo, entre los indicadores que miden los objetivos: el porcentaje de ciudadanos que piensan que la ciencia y la tecnología aportan más beneficios que perjuicios. El \% ha crecido del $47 \%$ al $61 \%$ desde 2002 a 2018, pero ha crecido mucho menos entre las personas con sin estudios o primarios. Está en el $39 \%$ en personas sin estudios frente al $76 \%$ de las personas universitarias. Entre medias, $47 \%$ con estudios primarios, $56 \%$ secundaria primer ciclo y $63 \%$ secundaria segundo ciclo.

Si tenemos en cuenta la población española total sin estudios superiores es el $69 \%$, (47\% que han cursado hasta la primera etapa de educación secundaria), la percepción de que la ciencia es más perjudicial que beneficiosa parece estar muy presente en la sociedad. Ante eso, el documento cita, tras las tablas con el indicador que "Teniendo en cuenta los datos reflejados anteriormente, el Eje 14 propone Promover el espíritu crítico y el compromiso de la sociedad española con la $1+D+1$, fomentando el equilibrio de género en investigación e innovación, la cultura científica, la reflexión y la decisión, en base a la evidencia científica, el fomento de ciencia y la innovación, e implementando todas ellas de forma abierta e inclusiva".

Sin duda estas cuestiones pueden ayudar, pero no deja de ser remarcable la ausencia total de menciones a las consecuencias indeseadas de la innovación, que pueden mejorar con una ciencia más inclusiva. Cabe preguntarse si esto es suficiente y aque sería necesario que se tengan presentes y que se dialogue sobre ellas. Parece que el foco de la estrategia es otro, en el apartado de resultados se observa que: "La mejora del impacto social de la investigación y la innovación. Se logrará abordando los retos de nuestra sociedad, favoreciendo la recuperación del país en base a la generación de conocimientoy de su transferencia. Es este sentido será fundamental que la ciudadanía perciba la $I+D+l$ como un elemento esencial para mejorar su calidad de vida y la del entorno en el que habitan, implicándose activamente en mejorar sus conocimientos en ciencia e innovación." Es decir, se sitúa la I+D+I como un elemento esencial para mejorar la calidad de vida. 


\subsection{Monitorización: El Sistema de Información sobre Ciencia, Tecnología e Innovación}

Como se citaba anteriormente, se observa un lugar prioritario para el Sistema de Información sobre Ciencia, Tecnología e Innovación, el instrumento de monitorización y captación de datos que pretende alimentar las evaluaciones y desarrollos de la Estrategia y los citados Planes, así como es citado como un elemento clave de coordinación entre los diferentes niveles de sistema de Ciencia e Innovación. Es enmarcado como un elemento fundamental en la gobernanza y esta relevancia está muy presente en los documentos analizados y en la web del Ministerio actualmente.

La presencia de aspectos relacionados con RRI es no obstante testimonial. No se observa concordancia entre los objetivos enunciados en los textos y la monitorización, al menos en lo que atañe a retos sociales, ética, ciencia ciudadana y participación pública. Los aspectos que están presentes- si bien no siempre de forma directa- son género y acceso abierto.

Se observa a continuación un detalle del sistema de información y monitorización en relación a los documentos consultados.

\subsubsection{Antecedentes: seguimiento de las estrategias y planes}

En la web del Ministerio es posible observar en el apartado de gobernanza" que "el seguimiento de la Estrategia Española de Ciencia, Tecnología e Innovación se apoyará en cuatro instrumentos importantes:

- El Sistema de Información de Ciencia, Tecnología e Innovación (SICTI).

- PAID -Plataforma Automatizada de Datos de I+D+i- que se integra en el primero.

- La Red de Políticas Públicas de I+D+l que sirve de foro de intercambio de información y experiencias entre todos los agentes (AGE y Comunidades Autónomas).

- ICONO -Observatorio Español de I+D+i- que coordinado por la Secretaría de Estado de Investigación, Desarrollo e Innovación y junto con los observatorios específicos existentes a nivel regional analizará las tendencias, coordinará los estudios necesarios en materia de impacto de los resultados y prospectiva de tendencias y necesidades, para el seguimiento de la Estrategia Española de Ciencia, Tecnología e Innovación y los planes que la desarrollan."

Con respecto a ICONO, está gestionado por la Fundación Española para la Ciencia y la Tecnología (FECYT) e incluye indicadores de género y cultura científica (percepciones sociales sobre la ciencia y la tecnología, no se incluyen relativos a participación ciudadana o ciencia ciudadana), además de diversos indicadores relacionados con recursos humanos, inversión, alta tecnología, TIC, internacionalización, transferencia o biotecnología. También existe un epígrafe relacionado con indicadores socioeconómicos, si bien están centrados en la medida PIB en su totalidad.

\subsubsection{Primera estrategia}

Por su parte, el documento de la Estrategia 2013-2020 plantea indicadores concretos de medición. Estos indicadores se centran en el control de los recursos y la eficiencia, para observar

7

https://www.ciencia.gob.es/portal/site/MICINN/menuitem.8ce192e94ba842bea3bc811001432ea0/?vg nextoid $=8 \mathrm{e} 5 \mathrm{db} 1 \mathrm{c} 445673410 \mathrm{VgnVCM} 1000001 \mathrm{~d} 04140 \mathrm{aRCRD \# :} \mathrm{:text=PAID \% 20 \% E2 \% 80 \% 93Plataforma \%}$ 20Automatizada\%20de\%20Datos,(AGE\%20y\%20Comunidades\%20Aut\%C3\%B3nomas). Visitado el 7 de agosto de 2020. 
el grado de compromiso de los actores, especialmente en términos de inversión, así como para observar los aumentos en el grado de formación y contratación de capital humano I+D+i y en las publicaciones y patentes. No se presenta ni se enmarca como un elemento de transparencia o de gobierno abierto, ni se citan las posibles distorsiones de la métrica (y sus consecuencias no deseadas). La métrica no incluye ninguno de los aspectos relacionados con los aspectos mencionados de la RRI (desafíos sociales, igualdad de género, acceso abierto, etc.), aunque existe un indicador relacionado con la educación para la ciencia "Evolución del valor social de la ciencia y la tecnología", que se establece como positivo a priori, siguiendo la orientación general de la estrategia.

\subsubsection{Planes 2013-2020}

Los planes también contemplan indicadores específicos. Los indicadores 2013-2016 se ciñen a lo planteado en la estrategia, mientras en el plan 2017-2020 se observa un mayor despliegue.

Se definen indicadores de gestión, divididos en indicadores relativos a los órganos convocantes de las convocatorias, e indicadores referidos a las propias convocatorias, fundamentalmente orientados las cantidades liberadas, distribución geógrafica etc. Podrían definirse como un mapa de los fondos y sus solicitantes.

Por su parte, los indicadores relativos a objetivos y resultados del Plan 2017-2020, se indica que "Estos indicadores han sido identificados a partir de: (1) los objetivos específicos de cada uno de los Programas y Subprogramas; (2) la coherencia entre las actuaciones propuestas y los indicadores a seleccionar; (3) la disponibilidad y accesibilidad de los indicadores y de la información que permite su construcción; (4) los resultados perseguidos con las actuaciones incluidas en el Plan Estatal. (5) los indicadores incluidos en el Programa Operativo de Crecimiento Inteligente 2014-2020 correspondientes al Objetivo Temático 1 que están asociados a las actuaciones del presente Plan Estatal"

Además de indicadores relativos a esfuerzo (fundamentalmente inversión), se incluyen indicadores que miden cada uno de los 4 programas estatales anteriormente definidos. Así, el programa relativo a recursos humanos incide en el número de doctores y su tasa de ocupación, así como el número de doctores contratados directamente a través de las ayudas programadas por el Plan. El programa de generación de conocimiento y fortalecimiento científico y tecnológico mide el número de publicaciones científicas en colaboración internacional, participación en programas de financiación europeos (ERC, H2020), patentes, proyectos de I+D+I generados en universidades y centros públicos de investigación con financiación empresarial, etc. Se incluye aquí el indicador relativo al número de publicaciones en acceso abierto. El programa de desarrollo empresarial se centra igualmente en indicadores de inversión y generación de proyectos. En materia de RRI cabría encontrar indicadores relacionados en el programa estatal dedicado a los retos sociales, sin embargo, los indicadores son muy similares respecto al programa de generación de conocimiento (publicaciones, colaboración internacional, captación de fondos europeos...). No hay ninguna referencia a cuestiones destacadas como clave en los textos del programa, como la interdisciplinariedad, la relevancia de la imbricación de las ciencias sociales y humanidades en otros retos sociales, o la participación pública que es citada en numerosas ocasiones.

\subsubsection{Nueva Estrategia 2021-2027}

Al igual que en anteriores estrategias y planes, el Sistema de Información se sitúa como un actor más de la gobernanza. La necesidad de seguimiento y evaluación es citada de forma continuada, y su espacio en el informe no se sitúa en la sección de gobernanza, sino en una sección dedicada 
específicamente a este aspecto, contando con su propio peso en la estructura de redacción de la estrategia.

En los indicadores definidos como prioritarios para el seguimiento de la estrategia, el único indicador relacionado con RRI es el número de mujeres investigadoras, que se espera que crezca desde el 38,8\% de 2018 al $42 \%$ en 2027 , si bien en la parte relativa a innovación o personal investigador en empresas no se desglosa por género pese a que la presencia femenina es considerablemente peor que en ciencia/ámbito público (más información en apartados posteriores). Los indicadores siguen la línea anterior, siendo su foco la excelencia y producción científica trasladada a indicadores que miden el \% de artículos publicados en revistas calificadas en el primer cuartil (Q1) y el $10 \%$ más citados, proyectos europeos y su retorno, así como datos de recursos humanos e inversión efectuada.

Sin embargo, en el Anexo es posible encontrar indicadores asociados a objetivos y ejes de la estrategia donde aparecen indicadores de interés en el marco RRI, bajo el título innovación y sociedad relativos a lo siguiente: número de estructuras de difusión de resultados a la sociedad, percepción social de que la ciencia aporta más beneficios que perjuicios, y número de comités que incluyen asesoramiento de la sociedad civil. Se observa por tanto un interés en observar la participación de la sociedad en la ciencia no sólo en el ámbito comunicativo unidireccional, a diferencia de los anteriores documentos. Así mismo, se observa un indicador dedicado a multidisciplinariedad.

Como curiosidad, en el apartado de gobernanza aparece un indicador que medirá el número de nuevos indicadores disponibles en el sistema de información, lo cual no deja de ser llamativo ya que el número de nuevos indicadores no necesariamente orienta con respecto a su propósito, la utilidad o posible uso en el propio sistema de gobernanza.

Tabla 5. Indicadores relacionados con RRI (disponibles en Anexo, seguimiento de Objetivos):

Ind. 3.1. Esfuerzo presupuestario en I+D de los departamentos ministeriales.(SICTI)

Ind. 4.4. Interés social por la ciencia. (FECYT)

Ind. 5.2. Porcentaje de personal empleado en actividades de I+D mujeres.(INE)

Ind. Eje. 7.4. Porcentaje de mujeres investigadoras. (Eurostat/INE)

Ind. Eje. 7.5. Porcentaje de mujeres que son primer autor en una publicación.(FECYT)

Ind. Eje 10.1 Porcentaje de proyectos del PEICTI que son evaluados en

comisiones multidisciplinares. (AEI/CDTI/ISCIII)

Ind. Eje 14.1 Número de entidades del SECTI que cuentan con estructuras

de difusión a la sociedad del conocimiento científico. (FECYT)

Ind. Eje 14.2 Porcentaje de la población que opina que los beneficios de la ciencia y la tecnología son mayores que sus perjuicios. (FECYT).

Ind. Eje. 14.3. Número de comités de asesoramiento que incluyen a la sociedad civil. (MICIN) 
Cabe situar dos ausencias destacadas: indicadores relacionados con responsabilidad que apliquen al sector empresarial e indicadores de ciencia abierta, pese a lo presente que está en el documento y sus últimos desarrollos a nivel europeo ${ }^{8}$.

\subsubsection{Otras iniciativas de monitorización relacionadas con RRI: género}

Existen sin embargo otras iniciativas de monitorización relacionadas con aspectos RRI, como es el caso de los informes de género emitidos por el Observatorio de Mujer y Ciencia. En el ámbito científico se desarrollan desde 2007, mientras en el ámbito de innovación se desarrolló el primer informe en el año 2020, señalando la ausencia de datos dada la no desagregación por sexo y la carencia de información de contexto y relativa a las personas que participan en la innovación en las encuestas de innovación, tanto internacionales como nacionales ${ }^{9}$. Los escasos resultados disponibles muestran una situación notablemente peor que en el ámbito de ciencia, con una proporción de mujeres muy escasa en empresas innovadoras financiadas con fondos públicos, entre el $18-23 \%$, así como peores resultados en liderazgo o transferencia.

Sin embargo, los indicadores relativos a género no se encuentran en el centro del sistema de gobernanza, como se señalaba, no se encuentran entre los indicadores del sistema definido en la web del Ministerio. Son mediciones periféricas, no hay indicaciones definidas en los documentos estratégicos para canalizarlas e integrarlas en las políticas.

Esta situación cambia ligeramente en la nueva Estrategia Española de Ciencia, Tecnología e Innovación 2021-2027. En el DAFO se sitúa la presencia de mujeres científicas como una fortaleza al compararlo con la media europea, así como se incluye un indicador al respecto en el seguimiento de objetivos e indicadores básicos del sistema. Con respecto al área de innovación, en peor situación que la ciencia, se mide el \% de mujeres empleadas en actividades de I+D. Como nota, es posible que mediante el uso de estas mediciones no se refleje la gran diferencia que hay entre el sector público y privado en la presencia de mujeres en este ámbito ${ }^{10}$.

De manera general, podría discutirse situar como fortaleza la situación de las mujeres científicas. Si bien es más alta que la media europea, es una mala situación en absoluto para las mujeres del país ya que sigue habiendo desigualdad. Podría situarse como oportunidad, incluso como debilidad, atendiendo a que el foco sea la situación de las mujeres y no la comparativa internacional.

8 Comisión Europea (2020). Progress on Open Science: Towards a shared research knowledge system. Final Report on the Open Science Policy Platform". Bruselas, CE.

9 Más información en el informe "Mujeres e Innovación 2020", o la serie "Científicas en Cifras", publicados por el Observatorio de Mujeres, Ciencia e Innovación, Ministerio de Ciencia e Innovación.

${ }^{10}$ Consultar las recomendaciones del citado informe “Mujeres e Innovación 2020". 


\section{Conclusiones}

El análisis realizado de los documentos de gobernanza estratégica de la Ciencia, Tecnología e Innovación en España, desde el año 2014 hasta el presente período que alcanza hasta el año 2027, permite destacar dos aspectos en términos del marco utilizado en relación a una Ciencia e Innovación más responsable (RRI).

El primer lugar, se destaca la evolución observada entre el primer período 2014-2020, centrado en la competitividad y sin significativas referencias a aspectos sociales o relativos a responsabilidad, con respecto al segundo gran período 2021-2017, donde aspectos relacionados con la sostenibilidad, impacto social y participación pública están muy presentes en la nueva estrategia que inaugura la nueva programación. El contexto de la primera estrategia era la Gran Depresión iniciada en 2007-08, que en España alcanzó una mayor crudeza en los años 20122013- período en que se estaba elaborando el documento- coordinado por un gobierno situado a la derecha en el espectro político (Partido Popular). En el caso del nuevo período, la crisis pandémica- sanitaria, económica y social- ha sido planteada para justificar la mayor relevancia que la ciencia y la innovación deberían tener en la sociedad y en el conjunto de políticas públicas, incidiéndose en la transversalidad de la innovación en otras políticas, así como en la valoración científica en la toma de decisiones relevantes. Un gobierno de coalición situado a la izquierda ha promovido la nueva estrategia (Partido Socialista Obrero Español-PSOE, Unidas Podemos).

El contexto y la orientación política son factores de influencia notables, si bien cabe destacar la influencia de las políticas europeas en materia de RRI que se desprende del análisis documental realizado. En el último plan estatal (2017-2020) elaborado por el gobierno del Partido Popularlos planes desarrollan y hacen tangibles las estrategias- se observa cómo el vocabulario relacionado con RRI comienza a estar presente, coincidiendo con el desarrollo de las políticas europeas: acceso abierto, género y comunicación para la ciencia en especial. Podría citarse este caso como un nuevo ejemplo de mimetismo o isomorfismo institucional, en el que las políticas europeas se trasladan al contexto español sin especificar dimensiones, problemáticas o soluciones propias partiendo del marco europeo. El mimetismo también se observa en el nuevo período, si bien la presencia de elementos relacionados con responsabilidad en la estrategia es mucho más intensa, pudiéndose destacar este mayor grado como un rasgo particular que no necesariamente es traducción directa de lo que llega de Europa. En cualquier caso, la elaboración de las estrategias, que al mismo tiempo constituyen la Estrategia de Especialización Inteligente a nivel estatal, ha sido establecido por las autoridades europeas como un requisito o factor de gran relevancia para la recepción de fondos europeos de diversa índole, desde los fondos estructurales al proyectado fondo de recuperación para la pandemia. Esta situación de condicionalidad puede situarse posiblemente como uno de los grandes factores de influencia en el citado mimetismo con respecto a las políticas europeas de RRI.

Las estrategias, planes estatales y planes anuales son instrumentos de gobernanza que derivan de la compleja gobernanza europea, y que se ven influenciados por ésta en otro de sus particulares, como es la monitorización y seguimiento. En este caso se observa igualmente una evolución hacia el desarrollo de indicadores relacionados con aspectos RRI como sería el caso de la participación social o aspectos de género, si bien se asocian al cumplimiento de objetivos de la estrategia, mientras no están entre los indicadores clave, en el corazón de los indicadores señalados de capital importancia para su seguimiento. En este sentido, la propia estrategia cita que será necesario un mayor desarrollo, por lo que convendría una atención a estos aspectos en los futuros planes que se implementen. Destaca por el momento la ausencia de indicadores relacionados con Ciencia Abierta- aspecto sin embargo muy citado en la estrategia y con gran 
desarrollo a nivel europeo-así como la ausencia de indicadores relativos a responsabilidad que estén dirigidos al ámbito empresarial, ámbito clave en la estrategia especialmente en cuanto a transferencia de conocimiento e innovación. Otras áreas de las políticas públicas han hecho en estas materia grandes avances que podrían ser valorados para futuros desarrollos, como los paneles de indicadores existentes derivado de las políticas de Responsabilidad Social Corporativa. Diversos proyectos europeos han trabajado estos desarrollos en materia de innovación y ámbito empresarial y podrían ser tenidos en cuenta como es el caso del proyecto PRISMA.

En segundo lugar, cabe destacar cómo de manera global, se entiende el marco RRI en los documentos estratégicos, al margen de su evolución temporal. En este sentido se destacaría en todos ellos nuevamente la influencia de las políticas europeas con la aplicación del marco de áreas de trabajo o "keys" definido por la Comisión Europea y citado en la introducción del presente trabajo: género, gobernanza, ciencia abierta, educación para la ciencia, ética, etc, mientras el marco más académico enfocado en dimensiones como la anticipación o la reflexividad, está prácticamente ausente. Por último, cabe subrayar la total ausencia de referencias a las posibles consecuencias indeseadas o negativas de la innovación y la ciencia, aspecto que es especialmente relevante en el surgimiento del enfoque RRI.

En el documento de la última estrategia tampoco existen este tipo de referencias, así como no hay referencias explícitas al posible rechazo social a las consecuencias indeseadas de la innovación o a una distribución desigual de sus beneficios. Sin embargo, en el Anexo donde se hayan datos e indicadores con los que se ha elaborado la estrategia, se puede observar datos de opinión de los españoles y españolas con respecto a si la ciencia y la innovación son más beneficiosas que perjudiciales: las percepciones son más negativas que positivas en el caso de las personas sin estudios (sólo el 39\% lo percibe como más beneficioso), mientras la aprobación es claramente mayoritaria en personas con estudios superiores. Si tenemos en cuenta que la población española total sin estudios superiores es el $69 \%$ atendiendo a los últimos datos del INE consultados en julio de 2020 , (de ellos, el $47 \%$ han cursado hasta la primera etapa de educación secundaria), la percepción de que la ciencia es más perjudicial que beneficiosa parece estar muy presente en la sociedad. Ante eso, el documento cita, tras las tablas con el indicador: "Teniendo en cuenta los datos reflejados anteriormente, el Eje 14 propone Promover el espíritu crítico y el compromiso de la sociedad española con la $1+D+1$, fomentando el equilibrio de género en investigación e innovación, la cultura científica, la reflexión y la decisión, en base a la evidencia científica, el fomento de ciencia y la innovación, e implementando todas ellas de forma abierta e inclusiva".

Las referencias son muy escasas, pero podría comprenderse que el problema se enfoca en las percepciones de a quién están llegando los beneficios de la innovación, interpretando que puede que no estén llegando al conjunto de la sociedad y por ello se apela a la inclusividad. Este puede ser sin duda una de los factores que explican una percepción social que no es tan positiva, pero también cabría citar que la presentación de la ciencia y la innovación como aspectos netamente positivos puede estar ocultando otra posible faceta de la problemática, la relativa a las consecuencias inesperadas o negativas ya citadas, que también pueden contar con un impacto desigual en la sociedad.

Finalizando las conclusiones, destacar que se han analizado los marcos de partida, las miradas que nos indican cómo se entiende y se pone en perspectiva la política de RRI en España. También hemos citado algunos factores clásicos de influencia en las políticas públicas (color político del gobierno, contexto socio-económico, procesos de europeización) que nos muestran algunas posibles motivaciones de la orientación de los marcos observados. Esperamos que estos apuntes puedan ser útiles para futuras investigaciones, que podrían confirmar dichas motivaciones. Así mismo, las políticas de RRI en España se encuentran en una fase inicial de desarrollo, por lo que 
confiamos en que el análisis de los marcos de partida realizado pueda servir de base en futuros trabajos que exploren la implementación de las políticas dichas políticas, así como sus posibles resultados e impactos.

\section{Bibliografía}

- European Commission. (2012). Responsible Research and Innovation: Europe's Ability to Respond to Societal Challenges; European Union: Brussels, Belgium, 2012.

- European Commission.(2014). Responsible Research and Innovation: Europe's Ability to Respond to Societal Challenges;European Union: Brussels, Belgium, 2014

- DiMaggio, P. J., and W. W. Powell. 2000. "The Iron Cage Revisited Institutional

- Isomorphism and Collective Rationality in Organizational Fields." In Economics

- Meets Sociology in Strategic Management 17: 143-166.

- Grinbaum, A., \& Groves, C. (2013). What is "responsible" about responsible innovation? Understanding the ethical issues. Responsible innovation: Managing the responsible emergence of science and innovation in society, 119-142.

- Hagendijk, R., \& Irwin, A. (2006). Public deliberation and governance: engaging with science and technology in contemporary Europe. Minerva, 44(2), 167-184.

- Jasanoff, S. (2015). Future imperfect: Science, technology, and the imaginations of modernity. Dreamscapes of modernity: Sociotechnical imaginaries and the fabrication of power, 1-33.

- Jonas, H. (1995). El principio de responsabilidad: ensayo de una ética para la civilización tecnológica. Herder. Original 1979 Das Prinzip Verantwortung. Versuch einer Ethik für die technologische Zivilisation, Frankfurt am Main

- Mackay, F., Monro, S., \& Waylen, G. (2009). The feminist potential of sociological institutionalism. Politics \& Gender, 5(2), 253-262.

- Mejlgaard, N., Woolley, R., Bloch, C., Bührer, S., Griessler, E., Jäger, A., ... \& Peter, V. (2018). A key moment for European science policy. Journal of Science Communication, 17(3) https://doi.org/10.22323/2.17030305

- Nielsen, M. V., Lindner, R., Bryndum, N., Burchardt, U., Schofield, M., \& Stilgoe, J. (2016). Navigating towards Responsible Research and Innovation: Challenges for Policy and Governance. In 2015 European Technology Assessment Conference in Berlin (pp. 5762).

- Owen, R., Bessant, J., \& Heintz, M. (2013). Responsible innovation: Managing the responsible emergence of science and innovation in society. John Wiley \& Sons

- Owen, R., Macnaghten, P., Stilgoe, J., \& others. (2012). Responsible research and innovation: From science in society to science for society, with society. Science and public policy, 39(6), 751-760.

- Owen, R., Stilgoe, J., Macnaghten, P., Gorman, M., Fisher, E., \& Guston, D. (2013). A framework for responsible innovation. Responsible innovation: managing the responsible emergence of science and innovation in society, 27-50.

- Stilgoe, J., Owen, R., \& Macnaghten, P. (2013). Developing a framework for responsible innovation. Research Policy, 42(9), 1568-1580.

- Von Schomberg, Rene. (2013). A vision of responsible research and innovation. Responsible innovation: Managing the responsible emergence of science and innovation in society, 51-74.

- Von Schomberg, René. (2014). The quest for the 'right'impacts of science and technology: a framework for Responsible Research and Innovation. En Responsible Innovation 1 (pp. 33-50). Springer. 


\section{Evolución de la perspectiva responsable en la Ciencia e Innovación (RRI) en España: un análisis de los documentos de gobernanza estratégica}

Paula Otero-Hermida

Diciembre 2020

Para citar este documento: Paula Otero-Hermida (2020). Evolución de la perspectiva responsable en la Ciencia e Innovación en España (RRI): un análisis de los documentos de gobernanza estratégica. December 2020, Working paper, SUPER_MoRRI project www.supermorri.eu 